\title{
Radio and X-ray diffuse emission in six clusters of galaxies
}

\author{
F. Govoni ${ }^{1,2}$, L. Feretti ${ }^{2}$, G. Giovannini ${ }^{2,3}$, H. Böhringer ${ }^{4}$, T. H. Reiprich ${ }^{4}$, and M. Murgia ${ }^{2,3}$ \\ 1 Dipartimento di Astronomia, Univ. Bologna, Via Ranzani 1, 40127 Bologna, Italy \\ 2 Istituto di Radioastronomia - CNR, via Gobetti 101, 40129 Bologna, Italy \\ 3 Dipartimento di Fisica, Univ. Bologna, Via B. Pichat 6/2, 40127 Bologna, Italy \\ 4 Max-Planck-Institut für Extraterrestrische Physik, PO Box 1603, 85740 Garching, Germany
}

Received 30 March 2001 / Accepted 6 July 2001

\begin{abstract}
Deep Very Large Array radio observations confirm the presence of halo and relic sources in six clusters of galaxies (A115, A520, A773, A1664, A2254, A2744) where a wide diffuse emission was previously found in the NRAO VLA Sky Survey. New images at $1.4 \mathrm{GHz}$ of these six clusters of galaxies are presented and X-ray data obtained from the ROSAT archive are analyzed. The properties of clusters hosting radio halos and relics are analyzed and discussed. A correlation between the halo radio power and the cluster gravitational mass is presented.
\end{abstract}

Key words. galaxies: clusters: general - intergalactic medium - magnetic fields - radio continuum: general X-rays: general

\section{Introduction}

An important problem in cluster phenomenology regards cluster-wide radio halos, whose prototype is Coma C (Willson 1970).

Radio halos in clusters of galaxies are diffuse radio sources located in the cluster center with no obvious connection to the galaxy population of the clusters. They are characterized by a typical size of about $1 \mathrm{Mpc}$, regular shape, low surface brightness and steep radio spectrum. Other similar sources, but in general with irregular morphologies, are named relics. They are found at the cluster periphery. In a few clusters, both a central halo and a peripheral relic are present.

These radio sources demonstrate the existence of relativistic electrons and large scale magnetic fields in the cluster intergalactic medium (IGM).

The study of these sources is very important since they are large scale features, which are related to other cluster properties in the optical and X-ray domain, and are thus directly connected to the cluster history and evolution.

Radio halos and relics have always been considered very rare structures. However, thanks to the sensitivity of the radio telescopes and to the existence of deep radio surveys, the number of known halos and relics has recently increased.

Giovannini et al. (1999), using the NRAO Very Large Array (VLA) Sky Survey (NVSS, Condon et al. 1998),

Send offprint requests to: F. Govoni,

e-mail: fgovoni@ira.bo.cnr.it searched for new radio halos and relics in the sample of X-ray-brightest Abell cluster (XBACs) presented by Ebeling et al. (1996).

The XBACs sample consists of clusters/subclusters from the catalogue of Abell et al. (1989) detected in the ROSAT All Sky survey (RASS) with X-ray flux $f_{\mathrm{X}}>$ $5 \times 10^{-12} \mathrm{erg} \mathrm{cm}^{-2} \mathrm{~s}^{-1}$ in the $0.1-2.4 \mathrm{keV}$ energy range. This is an all-sky, X-ray flux limited sample, complete in the galactic latitude range $|b| \geq 20^{\circ}$ and in the redshift interval $z \leq 0.2$, but it contains also 12 clusters at lower galactic latitude and 24 clusters with redshift greater than 0.2 that meet the flux criterion. The NVSS was performed at $1.4 \mathrm{GHz}$ with the VLA in D configuration. It has an angular resolution of $45^{\prime \prime}(F W H M)$, a noise level of $0.45 \mathrm{mJy} /$ beam $(1 \sigma)$ and covers all the sky north of Declination $=-40^{\circ}$. Because of the lack of short baselines, Giovannini et al. (1999) used as a limiting redshift for the search of diffuse sources the value $z \geq 0.044$. Considering this redshift limit, and taking into account the declination limit of the NVSS, they searched for diffuse emission in 207 clusters. They found 29 clusters, 11 of which were already known in the literature to possess a radio halo or relic, whereas 18 were new detections.

To properly map these diffuse sources high sensitivity to the extended features is needed, but also a good resolution is necessary to distinguish a real diffuse source from the blend of unrelated sources.

We started a project to study all these new radio halo and relic candidates with improved sensitivity and resolution with respect to the NVSS. Here we present the data 
Table 1. General properties of the clusters.

\begin{tabular}{lcccc}
\hline Name & $z$ & Richness & BM type & $\mathrm{kpc} /{ }^{\prime \prime}$ \\
\hline A115 & 0.1971 & 3 & III & 4.18 \\
A520 & 0.199 & 3 & III & 4.21 \\
A773 & 0.217 & 2 & II-III & 4.47 \\
A1664 & 0.1276 & 2 & & 3.00 \\
A2254 & 0.178 & 2 & & 3.88 \\
A2744 & 0.308 & 3 & III & 5.58 \\
\hline Col. 1: cluster name; Col. 2: redshift; Col. 3: richness class; \\
Col. 4: Bautz \& Morgan classification; \\
Col. 5: linear to angular conversion.
\end{tabular}

for the clusters A115, A520, A773, A1664, A2254, A2744 which were included in the first run of observations. The remaining clusters are presently being analyzed and will be presented in a future work.

The similarity between the radio and the X-ray morphology, the high cluster X-ray temperature and luminosity, and the evidence of substructures in clusters with radio halos (e.g. Feretti 1999) indicate the importance to analyze the X-ray properties of the cluster containing these diffuse sources. To obtain X-ray information and to find correlations between the radio and the X-ray parameters in clusters with radio halos and/or relics we analyzed pointed ROSAT observations for these clusters.

Here we present new radio images of these clusters, and compare them with the X-ray emission.

In Sect. 2 we present the radio and the X-ray data. In Sect. 3 we give the radio and the X-ray images. In Sect. 4 we perform an X-ray analysis, while the discussion and conclusions are reported in Sect. 5 .

We use a Hubble constant $H_{0}=50 \mathrm{~km} \mathrm{~s}^{-1} \mathrm{Mpc}^{-1}$ and $q_{0}=0.5$ throughout the paper.

\section{Data}

Some general properties of the six clusters of galaxies analyzed here are given in Table 1 . Deep radio images were obtained for all these clusters. The X-ray emission was analyzed using the data taken from the ROSAT public archive. The presence of sub-structures in X-ray images can give indications on the dynamical evolution of the clusters, however we also searched in literature for optical data indicating peculiar sub-structures in the galaxy distribution that could be interpreted in terms of cluster merging. As reported in the individual notes of the clusters, we found useful information for A115 and A520.

\subsection{Radio observations}

The radio data were obtained with the VLA at $1.4 \mathrm{GHz}$, in the $\mathrm{C}$ and $\mathrm{D}$ configurations for a total observing time of about 2 hours for each cluster in each configuration. The observing frequency and configurations were chosen in order to have a good sampling of short spacings,
Table 2. VLA observing log.

\begin{tabular}{ccccc}
\hline Name & $\begin{array}{c}\text { Frequency } \\
\text { MHz }\end{array}$ & $\begin{array}{c}\text { Bandw. } \\
\text { MHz }\end{array}$ & Config. & Date \\
\hline A115 & $1365 / 1435$ & 50 & C & December 98 \\
& $1365 / 1665$ & 50 & D & March 99 \\
A520 & $1365 / 1435$ & 50 & C & December 98 \\
& $1365 / 1665$ & 50 & D & March 99 \\
A773 & $1365 / 1435$ & 50 & C & November 98 \\
& $1365 / 1665$ & 50 & D & March 99 \\
A1664 & $1365 / 1435$ & 50 & C & November 98 \\
A2254 & $1365 / 1435$ & 50 & C & December 98 \\
& $1365 / 1665$ & 50 & D & April 99 \\
A2744 & $1365 / 1435$ & 50 & C & November 98 \\
& $1365 / 1665$ & 50 & D & March 99 \\
\hline
\end{tabular}

Col. 1: cluster name; Col. 2: observing frequency;

Col. 3: bandwidth; Col. 4: VLA configuration;

Col. 5: observing date.

Table 3. Primary flux density calibrators and phase calibrators.

\begin{tabular}{ccc}
\hline Name & Primary Cal. & Phase Cal. \\
\hline $\mathrm{A} 115$ & 3C48 & $0119+321$ \\
$\mathrm{~A} 520$ & 3C48 & $0459+024$ \\
$\mathrm{~A} 773$ & 3C286 & $0903+468$ \\
$\mathrm{~A} 1664$ & 3C286 & $1248-199$ \\
$\mathrm{~A} 2254$ & 3C286 & $1745+173$ \\
$\mathrm{~A} 2744$ & 3C48 & $0025-260$ \\
\hline
\end{tabular}

ensuring that a halo-type source could be easily detected and imaged.

The shortest baseline is $35 \mathrm{~m}$, corresponding to $\simeq 175 \lambda$. Therefore structures up to about $20^{\prime}$ in angular size are fully imaged. We note that our sources are all smaller in size. Only for A1664 where D array observations are not available we have a lack of short baselines which can affect our image of the relic radio source as discussed in Sect. 3.4.

The details of the radio observations are given in Table 2. The observations in D configuration were performed at distant frequencies within the same band, to obtain information on the spectral index of the diffuse sources.

The data were calibrated and reduced with the Astronomical Image Processing System (AIPS), following the standard procedure: Fourier-Transform, Clean and Restore. Self-calibration was applied to minimize the effects of amplitude and phase variations.

The source $0137+331$ (3C 48) or $1331+305$ (3C 286) was used as a primary flux density calibrator (see Table 3 ). The phase calibrator was observed every $20 \mathrm{~min}$, approximately.

We first reduced separately the data from the two different configurations, for a consistency check. Final 
Table 4. X-ray data from the ROSAT archive.

\begin{tabular}{cccccc}
\hline Name & Detector & $\begin{array}{c}t_{\text {exp }} \\
(\mathrm{s})\end{array}$ & $\begin{array}{c}\text { RA } \\
(\text { J2000.0 })\end{array}$ & $\begin{array}{c}\text { DEC } \\
(\text { J2000.0 })\end{array}$ & ROR \\
\hline A115 & HRI & 29692 & 005550.04 & 262436.0 & $800633 \mathrm{~h}-1$ \\
A520 & PSPC & 4850 & 045443.02 & 025212.0 & $800480 \mathrm{p}$ \\
A773 & HRI & 16663 & 091752.08 & 514348.0 & $800618 \mathrm{~h}$ \\
A1664 & PSPC & 12760 & 130343.02 & -241500.0 & $800411 \mathrm{p}$ \\
A1664 & HRI & 22227 & 130343.02 & -241500.0 & $800749 \mathrm{~h}$ \\
A2254 & HRI & 21976 & 171750.0 & 194012.0 & $800882 \mathrm{~h}-1$ \\
A2744 & PSPC & 13648 & 001419.02 & -302324.0 & $800343 \mathrm{p}$ \\
\hline
\end{tabular}

Col. 1: cluster name; Col. 2: ROSAT detector; Col. 3: exposure time;

Cols. 4, 5: pointing position; Col. 6: Rosat Observation Request Sequence Number.

Table 5. Radio parameters.

\begin{tabular}{ccccccccc}
\hline Name & type & $\begin{array}{c}S_{1400} \\
\text { mJy }\end{array}$ & $\begin{array}{c}\text { LLS } \\
\text { Mpc }\end{array}$ & $\alpha$ & $\begin{array}{c}P_{1400} \\
\text { Watt/Hz }\end{array}$ & $\begin{array}{c}P_{\text {tot }} \\
\text { Watt }\end{array}$ & $\begin{array}{c}\text { Volume } \\
\mathrm{Mpc}^{3}\end{array}$ & $\begin{array}{c}H_{\text {eq }} \\
\mu \mathrm{G}\end{array}$ \\
\hline $\mathrm{A} 115$ & $\mathrm{R}$ & 14.7 & 2.5 & 1.1 & $2.7 \times 10^{24}$ & $3.1 \times 10^{34}$ & 0.33 & 0.6 \\
$\mathrm{~A} 520$ & $\mathrm{H}$ & 34.4 & 1.4 & 1.7 & $6.4 \times 10^{24}$ & $4.0 \times 10^{35}$ & 0.42 & 1.3 \\
$\mathrm{~A} 773$ & $\mathrm{H}$ & 12.65 & 1.6 & 2.8 & $2.8 \times 10^{24}$ & $1.6 \times 10^{37}$ & 0.32 & 4.3 \\
$\mathrm{~A} 1664$ & $\mathrm{R}$ & 50.2 & 1.1 & $(1.2)$ & $3.7 \times 10^{24}$ & $5.2 \times 10^{34}$ & 0.17 & 0.8 \\
$\mathrm{~A} 2254$ & $\mathrm{H}$ & 33.7 & 1.2 & 1.2 & $4.9 \times 10^{24}$ & $7.0 \times 10^{34}$ & 0.40 & 0.7 \\
$\mathrm{~A} 2744$ & $\mathrm{H}$ & 57.1 & 2.3 & 1.4 & $2.6 \times 10^{25}$ & $6.3 \times 10^{35}$ & 3.40 & 0.8 \\
$\mathrm{~A} 2744$ & $\mathrm{R}$ & 18.2 & 2.0 & $>2$ & $8.5 \times 10^{24}$ & $>1.6 \times 10^{36}$ & 0.46 & $>1.9$ \\
\hline
\end{tabular}

Col. 1: cluster name; Col. 2: source type ( $\mathrm{R}=$ relic, $\mathrm{H}=$ halo); Col. 3: flux density;

Col. 4: largest linear size; Col. 5: average spectral index,

Col. 6: radio power at $1400 \mathrm{MHz}$; Col. 7: total radio power between $10 \mathrm{MHz}$ to $10 \mathrm{GHz}$;

Col. 8: radio source volume; Col. 9: equipartition magnetic field.

images were produced by adding together the C configuration data at 1365 and $1435 \mathrm{MHz}$, with the D configuration data at $1365 \mathrm{MHz}$. Images were produced with different resolutions, using the AIPS task IMAGR. The image with the highest available resolution $\left(\simeq 15^{\prime \prime}\right)$ provides information on the discrete radio sources, while the low resolution map $\left(\simeq 50^{\prime \prime}\right)$ allows the proper detection of the low brightness diffuse radio emission. All the data at $1665 \mathrm{MHz}$ are affected by interferences. A determination of the spectral index images is therefore difficult and only a total spectrum can be estimated.

\subsection{X-ray data}

We used archival observations of the 6 clusters of galaxies (A115, A520, A773, A1664, A2254, A2744) carried out with the Position Sensitive Proportional Counter (PSPC) and/or the High Resolution Imager (HRI) on ROSAT satellite.

The X-ray observations are identified by their Rosat Observation Request Sequence Number (ROR) in Table 4, where the detector, the exposure time, and the pointing position are also listed. When more than one observation existed for the same object with the same instrument, we used the observation with the longest exposure time.
The ROSAT energy band is $0.1-2.4 \mathrm{keV}$ for both detectors. The HRI provides a FWHM spatial resolution on axis $\simeq 5^{\prime \prime}$ and basically no spectral resolution, while the $\mathrm{PSPC}$ has a moderate angular resolution $\left(F W H M \simeq 25^{\prime \prime}\right.$ on axis), as well as spectral resolution. A detailed description of the instruments can be found in Trümper (1983) and Pfeffermann et al. (1987).

The data analysis has been performed with the EXSAS package (Zimmermann et al. 1994).

\section{Radio and X-ray images}

In the radio images presented here, the presence of diffuse sources is confirmed in all the six clusters of galaxies. In Table 5 we give the relevant parameters of the radio emission derived as follows:

1) The flux density was obtained after subtraction of the discrete sources, by integrating the surface brightness down to the noise level;

2) The radio spectral index was derived using the $\mathrm{D}$ configuration images at 1365 and $1665 \mathrm{MHz}$. Taking into account the closeness of the two frequencies, and some interferences in the images at $1665 \mathrm{MHz}$, this estimate of the spectral index should be taken cautiously with a typical error of about 0.5. For A1664, which lacks observation 

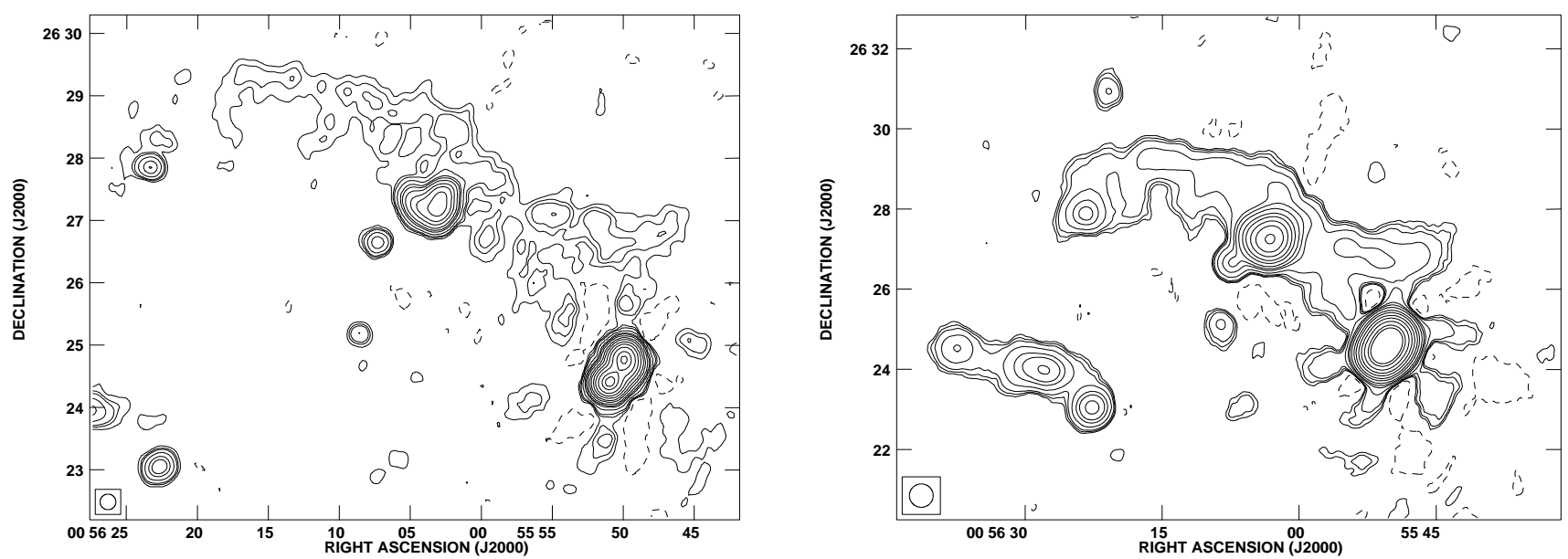

Fig. 1. Radio images at $1.4 \mathrm{GHz}$ of the relic source in A115. Left panel: the $F W H M$ is $15^{\prime \prime} \times 15^{\prime \prime}$; the noise level is $0.1 \mathrm{mJy} /$ beam. Contour levels are: -0.4 0.4 0.712461019325797169292400 mJy/beam. Right panel: the $F W H M$ is $35^{\prime \prime} \times 35^{\prime \prime}$; the noise level is $0.1 \mathrm{mJy} /$ beam. Contour levels are: -0.5 0.5 $0.712461019325797169292400 \mathrm{mJy} / \mathrm{beam}$. The pattern around the strong source $3 \mathrm{C} 28$ it is due to dynamic range problems. The peak of the image is coincident with this radio galaxy and it is $0.96 \mathrm{Jy} /$ beam.

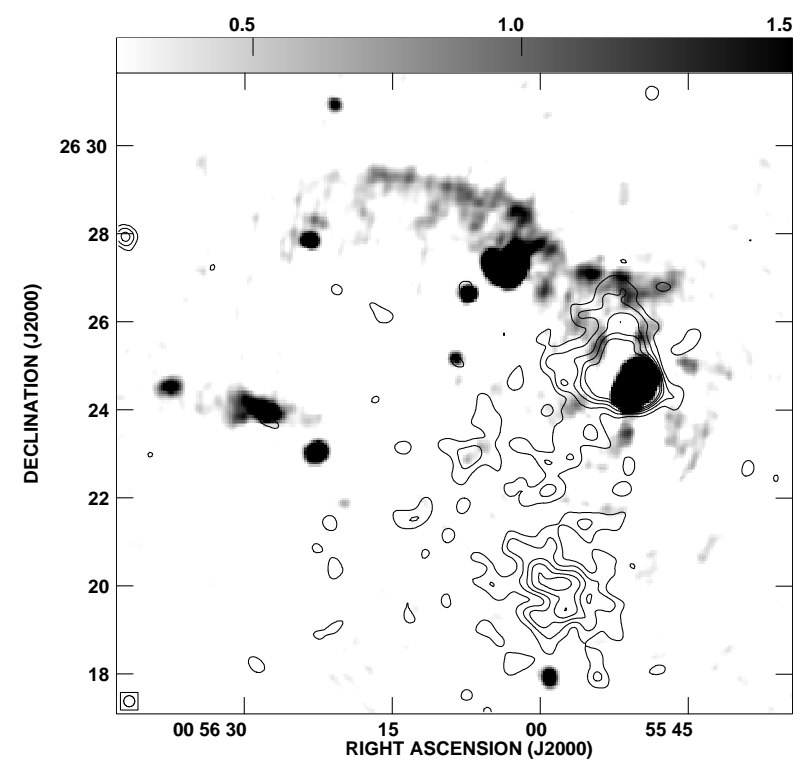

Fig. 2. Isophotal contour plots of the X-ray (HRI) image taken from the ROSAT archive, overlapped to the $1.4 \mathrm{GHz}$ greyscale image $\left(F W H M=15^{\prime \prime}\right)$ of the cluster A115. The X-ray

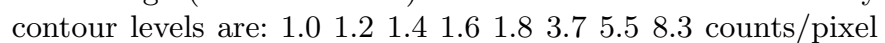
$\left(1\right.$ pixel $\left.=5^{\prime \prime} \times 5^{\prime \prime}\right)$.

at $1665 \mathrm{MHz}$, we assume $\alpha=1.2$, as found in typical relic radio sources (e.g. $1253+275$ in Coma);

3) The total power between $10 \mathrm{MHz}$ to $10 \mathrm{GHz}$ was derived using the spectral index given in the table;

4) The equipartition magnetic field was evaluated assuming a low frequency cutoff of $10 \mathrm{MHz}$, a high frequency cutoff of $10 \mathrm{GHz}$, equal energy density in protons and electrons, and a volume filling factor of 1 . The source volume was considered to be an ellipsoid.

The images of the X-ray brightness distribution presented in this work were produced by binning the photon events in a two-dimensional grid and then smoothing with a Gaussian filter. The PSPC images were produced in the hard band, corresponding to the energy range $0.5-2.0 \mathrm{keV}$. With the selection of this range of energies we minimize the Galactic and particle background (which dominate below $0.5 \mathrm{keV}$ and above $2.0 \mathrm{keV}$, respectively) while retaining most of the cluster emission. In the following we present the results and comment on each individual cluster.

\section{1. $A 115$}

The $1.4 \mathrm{GHz}$ radio images obtained with different angular resolutions are shown in Fig. 1, where the diffuse emission is easily visible.

The bright radio galaxy in the $\mathrm{SW}$ corner located at about $\mathrm{RA}(2000)=00^{\mathrm{h}} 55^{\mathrm{m}} 50^{\mathrm{s}}, \operatorname{DEC}(2000)=26^{\circ} 24^{\prime} 34^{\prime \prime}$ is $3 \mathrm{C} 28$. This source, showing here a double radio structure, has been studied in detail by Feretti et al. (1984) and Giovannini et al. (1987). In their images, this radio galaxy shows an unusual distorted morphology. It is characterized by two components located on both sides of the optical galaxy, with low brightness tails in the western direction, and without a clear radio compact core. The other strong source embedded within the diffuse emission is the radio source $0056+265$, a head-tail radio galaxy located at $\mathrm{RA}(2000)=00^{\mathrm{h}} 56^{\mathrm{m}} 03^{\mathrm{s}}, \operatorname{DEC}(2000)=$ $26^{\circ} 27^{\prime} 13^{\prime \prime}$ (Gregorini \& Bondi 1989). Both radio sources are associated with cluster galaxies.

The relic is elongated in the $\mathrm{SW}-\mathrm{NE}$ direction with a projected maximum extension of about $10^{\prime}(2.5 \mathrm{Mpc})$ and a transverse size about 5 times smaller. Other relic sources so extended are already known in the literature (see e.g. A3667, Röttgering et al. 1997).

In the higher resolution map, the Northern source boundary shows a sharper edge than on the opposite side. 

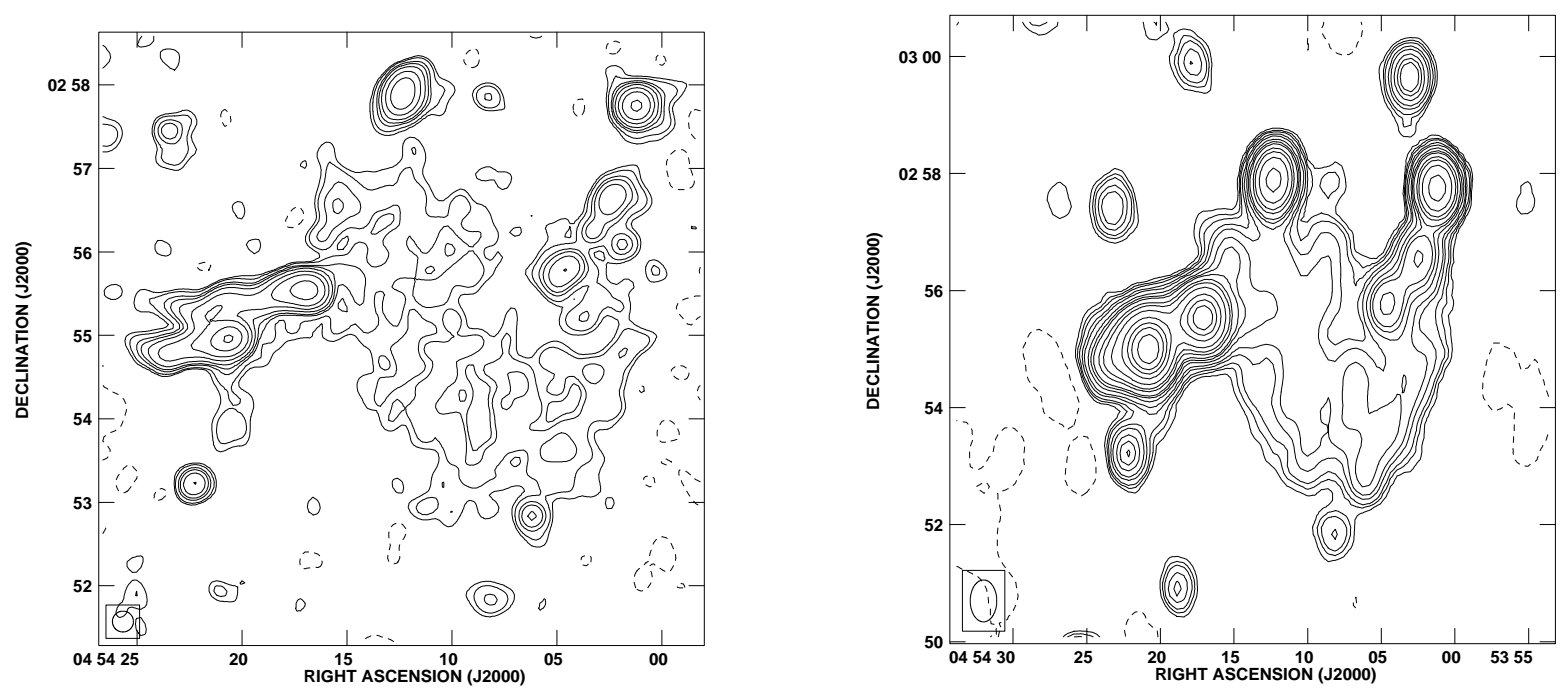

Fig. 3. Radio images at $1.4 \mathrm{GHz}$ of the halo source in A520. Left panel: the $F W H M$ is $15 \times 15^{\prime \prime}$; the noise level is $0.03 \mathrm{mJy} /$ beam. Contour levels are: - $0.060 .060 .10 .20 .30 .51251020 \mathrm{mJy} /$ beam. Right panel: the $F W H M$ is $43^{\prime \prime} \times 27^{\prime \prime}$; the noise level is $0.03 \mathrm{mJy} /$ beam. Contour levels are: -0.1 0.1 0.15 0.2 0.3 0.4 0.6 0.8 $11.523579121825 \mathrm{mJy} / \mathrm{beam}$.

A similar feature is seen in other relics (e.g. 1253+275 in the Coma cluster, Giovannini et al. 1991; A3667, Röttgering et al. 1997). This could be related to some compression of the plasma.

A115 is known in literature to be characterized by a double X-ray peak (Forman et al. 1981). The X-ray data at our disposal have been obtained with the ROSAT HRI. In Fig. 2 we show the X-ray image (contours), smoothed with a Gaussian of $\sigma=10^{\prime \prime}$ over-imposed onto the radio image (grey scale) at resolution of $15^{\prime \prime}$. In the figure, a double X-ray morphology is evident with two irregular enhancements in the X-ray surface brightness distribution. Part of the X-ray emission in the northern peak could come from the contribution of the radio galaxy 3C 28 .

The diffuse radio source belongs to the northern X-ray clump, and extends from the sub-cluster center to the periphery. According to its non-central location and its elongated structure, it is classified as a cluster relic. However, elongated relics, as for example those in Coma, A3667, A2255, are generally at the cluster periphery, and with the largest dimension roughly perpendicular to the cluster radial orientation. So this source is quite unusual, and difficult to interpret in the framework of currently proposed models, invoking spherically symmetric compression of the IGM by shock waves during the cluster formation (Enßlin et al. 1998; Roettiger et al. 1999). A possibility is that this source is actually in foreground or background with respect to the cluster center.

We have considered the possibility that this radio source could be part of a supernova remnant, but we have found no identification in the catalog of Green (2000). Inspection of a large field of the NVSS reveals no other diffuse radio structure, which could be part of the same supernova remnant. Moreover the radio spectrum found for this source is much steeper than typical supernova remnant spectra (Green 2000). Therefore this interpretation

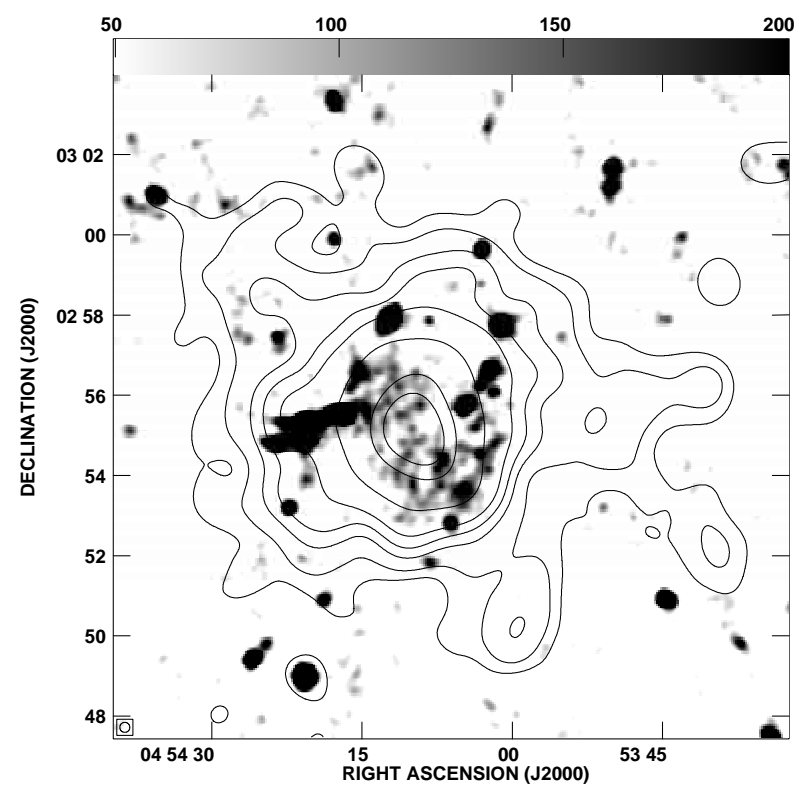

Fig. 4. Isophotal contour plots of the X-ray (PSPC) image taken from the ROSAT archive, overlapped to the $1.4 \mathrm{GHz}$ grey-scale image $\left(F W H M 15^{\prime \prime}\right)$ of the cluster A520. The X-ray contours are: 0.20 .30 .50 .71245 counts $/$ pixel $(1$ pixel $=$ $\left.15^{\prime \prime} \times 15^{\prime \prime}\right)$.

seems very unlikely, confirming that this source should be a cluster relic.

In the optical band, Beers et al. (1983) mapped the galaxy distribution of the cluster over a field of $3.2 \mathrm{Mpc}$ square. The galaxy distribution reveals the presence of three primary clumps of galaxies.

They further compared the X-ray image from Forman et al. (1981) with the optical galaxy distribution finding that the two main optical clumps correspond to the two peaks in the X-ray distribution. The third optical clump, located at $7^{\prime}$ to the east of the two main peaks, has no 

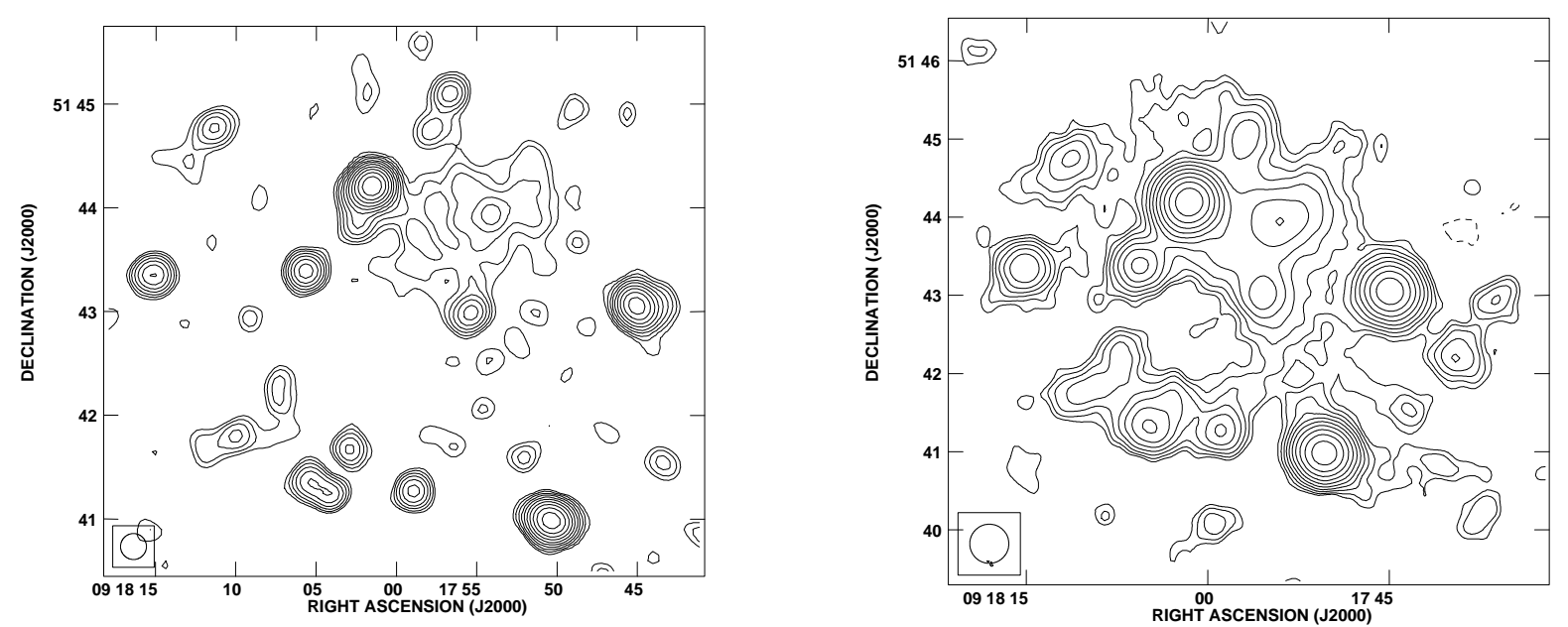

Fig. 5. Radio images at $1.4 \mathrm{GHz}$ of the halo source in A773. Left panel: the $F W H M$ is $15^{\prime \prime} \times 15^{\prime \prime}$; the noise level is $0.02 \mathrm{mJy} /$ beam. Contour levels are: -0.060 .060 .080 .120 .170 .240 .340 .480 .680 .961 .361 .922 .713 .845 .437 .68 10.86 mJy/beam. Right panel: the $F W H M$ is $30^{\prime \prime} \times 30^{\prime \prime}$; the noise level is $0.03 \mathrm{mJy} /$ beam. Contour levels are: -0.070 .070 .10 .140 .20 .280 .400 .560 .791 .12$ $1.582 .243 .174 .486 .348 .9612 .6717 .92 \mathrm{mJy} /$ beam.

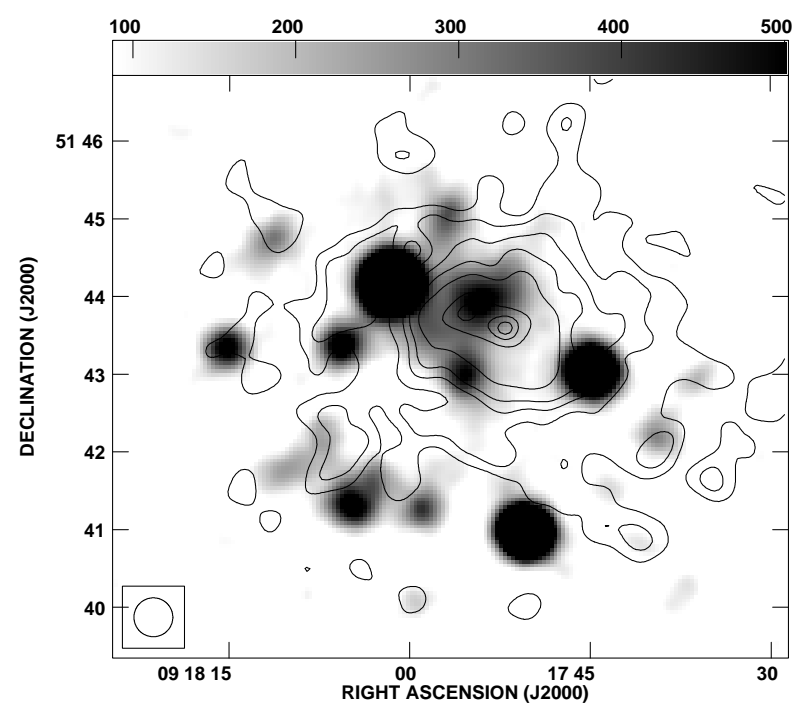

Fig. 6. Isophotal contour plots of the X-ray (HRI) image taken from the ROSAT archive overlapped to the grey-scale image at $1.4 \mathrm{GHz}\left(F W H M 30^{\prime \prime}\right)$ of the cluster A773. The X-ray contours are: 0.650 .811 .533 .43 .6 Counts $/$ pixel $\left(1\right.$ pixel $\left.=5^{\prime \prime} \times 5^{\prime \prime}\right)$.

X-ray emission detected. We note, however, that the third optical peak is located in coincidence of the radio elongated emission visible in the bottom left corner of the low resolution radio image at about $\mathrm{RA}(2000)=00^{\mathrm{h}} 56^{\mathrm{m}} 30^{\mathrm{s}}$, $\operatorname{DEC}(2000)=+26^{\circ} 24^{\prime}$, (Fig. 1, right panel).

\section{2. $A 520$}

The $1.4 \mathrm{GHz}$ images of the center of A520 are shown in Fig. 3. The radio halo shows a low surface brightness with a clumpy structure. The structure is slightly elongated in the NE-SW direction, with a maximum projected size of about $340^{\prime \prime}$ (1.4 Mpc).
There are several discrete radio sources at the boundary of the halo. The two head-tail radio sources located on the eastern side of the halo are identified with cluster galaxies. The projected extension is about $600 \mathrm{kpc}$ for the northern head-tail radio galaxy and about $400 \mathrm{kpc}$ for the southern one. It is remarkable the fact that the tails in these sources are oriented toward the same direction, opposite to the cluster center. According to Bliton et al. (1998) this could be related with bulk motion of the intergalactic medium and could therefore indicate the presence of a merger in this cluster. Alternatively they could both belong to the same infalling group.

The X-ray image of this cluster, obtained with the ROSAT PSPC, was produced by binning the photon events in a two-dimensional grid and then smoothing with a Gaussian filter of $\sigma=30^{\prime \prime}$. In Fig. 4 we present the $\mathrm{X}$-ray image (contours) of the cluster over-imposed onto the radio image (grey scale). The $\mathrm{X}$-ray brightness distribution shows structure, with the inner region elongated in the NE-SW direction, and the outer region elongated in a perpendicular direction (SE-NW) with an extension to the West only seen in the X-ray emission. The radio halo is located at the cluster center; its structure is elongated in the same direction as the central X-ray emission.

Proust et al. (2000) presented a study of the dynamics of A520. The cluster seems to be undergoing strong dynamical evolution, since its $\mathrm{cD}$ galaxy it is not located at the center of the galaxy distribution. Moreover they pointed out that this cluster may be an example of a dynamically young system where clumps of galaxies are still in phase of collapsing.

\section{3. $A 773$}

In Fig. 5 we show the radio images at $1.4 \mathrm{GHz}$ of the cluster A773, with two different angular resolutions. The halo is quite regular, with a total size of about $6^{\prime}(1.6 \mathrm{Mpc})$. 

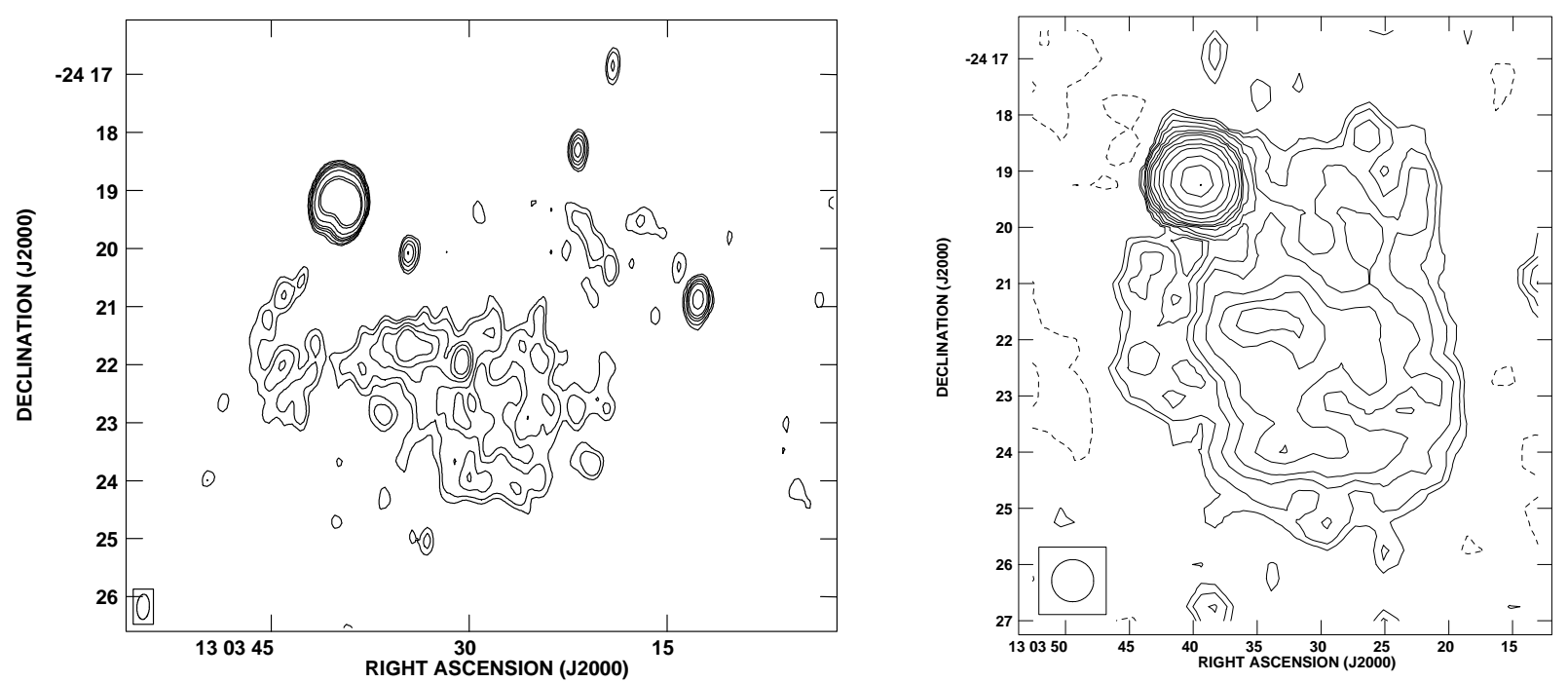

Fig. 7. Left panel: radio images of the relic source in A1664. The $F W H M$ is $26^{\prime \prime} \times 13^{\prime \prime}$; the noise level is $0.07 \mathrm{mJy} /$ beam. Contour levels are: $0.20 .30 .50 .71257 \mathrm{mJy} /$ beam. Right panel: radio image of A1664 taken from the NVSS. Contour levels are: -1 $0.711 .5234571015254055 \mathrm{mJy} /$ beam.

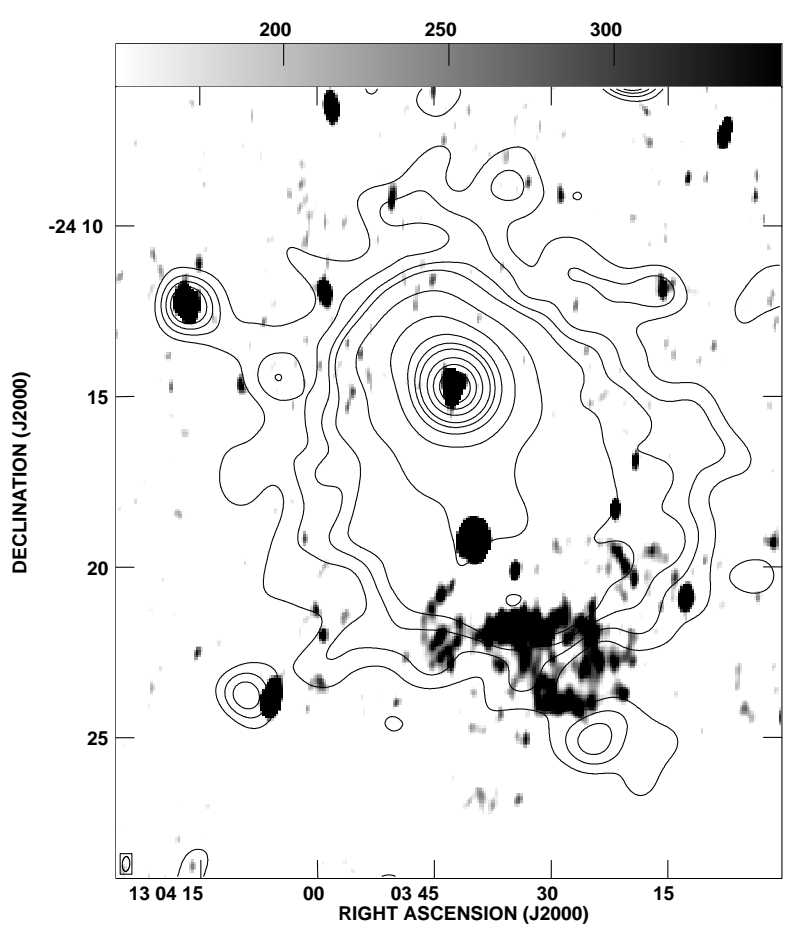

Fig. 8. Isophotal contour plots of the X-ray (PSPC) image taken from the ROSAT archive overlapped to the grey-scale image at $1.4 \mathrm{GHz}\left(F W H M 26^{\prime \prime} \times 13^{\prime \prime}\right)$ of the cluster A1664. The X-ray contours are: 0.40 .60 .812510152030405060 counts $/$ pixel $\left(1\right.$ pixel $\left.=15^{\prime \prime} \times 15^{\prime \prime}\right)$.

The brightness distribution of the extended emission is peaked in the center. However, in the VLA FIRST survey (Becker et al. 1995) no discrete source is present in the central halo region at a level of $0.3 \mathrm{mJy} /$ beam.

The higher resolution image shows many discrete sources. In particular it is evident that the elongated feature visible to the south in the low resolution map is due to a blend of individual sources.
The X-ray image (contours) presented in Fig. 6 is obtained with the ROSAT HRI, by smoothing with a Gaussian filter of $\sigma=10^{\prime \prime}$. The X-ray brightness distribution is very irregular with a clear elongation, which is probably due to the presence of two close peaks at the distance of about $30^{\prime \prime}(134 \mathrm{kpc})$. The radio halo is located at the cluster center, with the brightest region coincident with the eastern X-ray peak. The presence of X-ray substructures in A773 was also noted by Rizza et al. (1998).

This cluster has also been target for the study of the Sunyaev-Zeldovich effect. The temperature decrement detected toward A773 at $28.7 \mathrm{GHz}$ is $-575<\Delta T<$ $-760 \mu \mathrm{K}$ (Carlstrom et al. 1996).

\section{4. $A 1664$}

The $1.4 \mathrm{GHz}$ radio image presented in Fig. 7 (left panel) shows the diffuse source in detail. The source is located at the projected distance of about $6^{\prime}(1.08 \mathrm{Mpc})$ from the cluster center, and is therefore a relic. From this image we derive a flux density of $50.2 \mathrm{mJy}$. Due to the missing short spacings, some flux may be missed in our image. This is confirmed in the NVSS image (see Giovannini et al. 1999 and Fig. 7, right panel) where the structure appears much more extended, quite uniform and the calculated flux density is 107 mJy. No spectral index information is available for this cluster, therefore in the equipartition magnetic field strength and total power calculations we assumed a mean spectral index $\alpha=1.2$, as a typical value.

The ROSAT PSPC image (contours) for the cluster A1664, overlaid onto the radio image (grey-scale), is shown in Fig. 8. The X-ray image was smoothed with a Gaussian of $F W H M=30^{\prime \prime}$. The X-ray emission is asymmetric with an elongation toward the south direction, where the relic is located. Close to the X-ray emission center there is a strong radio source well visible in the image. This is 


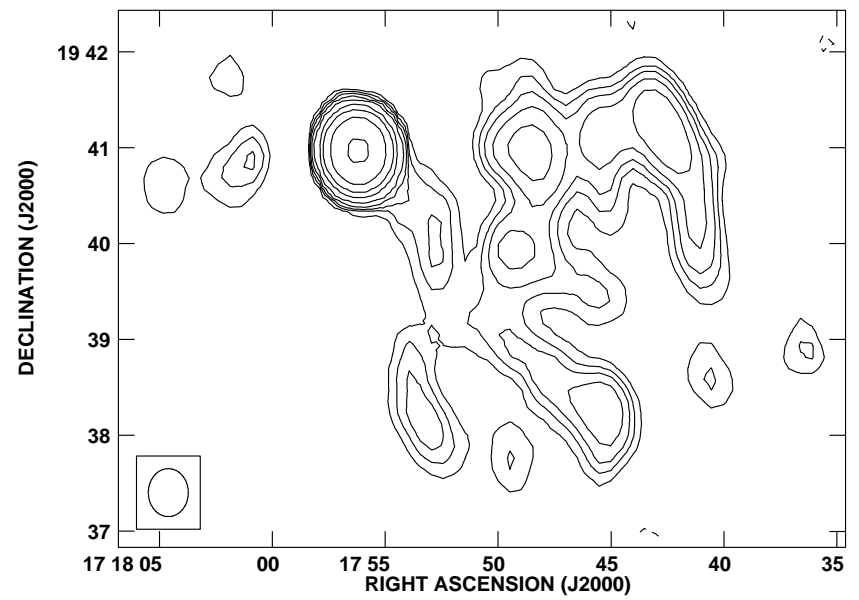

Fig. 9. Radio image at $1.4 \mathrm{GHz}$ of the halo source in A2254. The FWHM is $30^{\prime \prime} \times 25^{\prime \prime}$; the noise level is $0.1 \mathrm{mJy} /$ beam. Contour levels are: $-0.30 .30 .50 .711 .5351030 \mathrm{mJy} /$ beam.

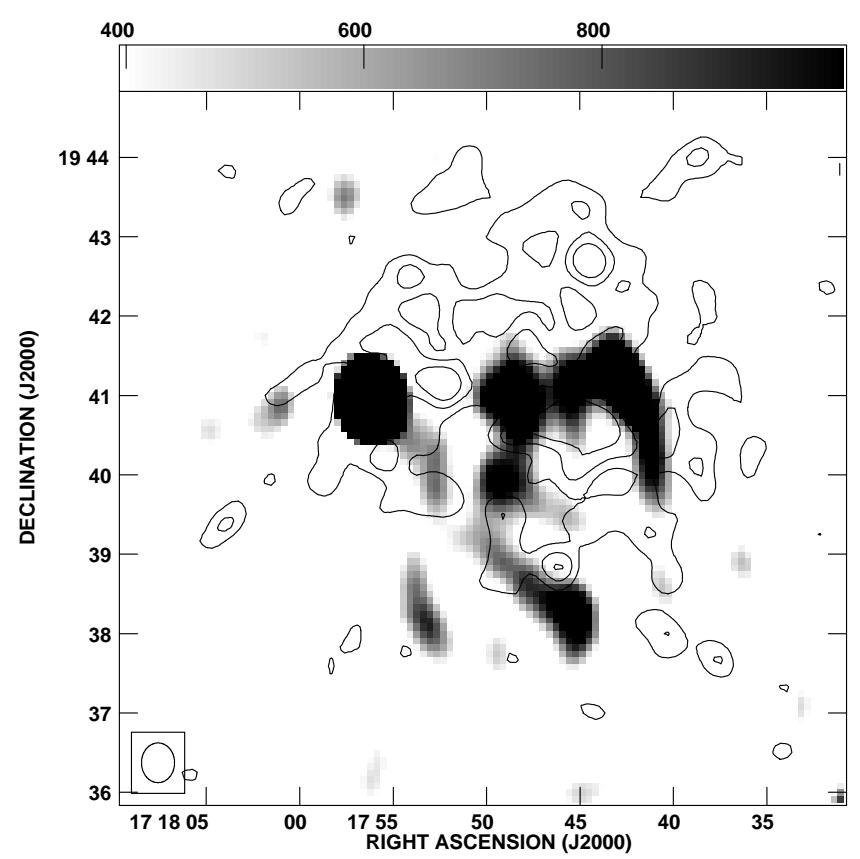

Fig. 10. Isophotal contour plots of the X-ray (HRI) image taken from the ROSAT archive overlapped to the greyscale image at $1.4 \mathrm{GHz}\left(F W H M 30^{\prime \prime} \times 25^{\prime \prime}\right)$ of the cluster A2254. The X-ray contours are: 1.11 .31 .522 .5 counts/pixel $\left(1\right.$ pixel $\left.=5^{\prime \prime} \times 5^{\prime \prime}\right)$.

identified with an optically line-luminous galaxy, studied by Allen et al. (1995). Other radio galaxies are located inside and to the North of the relic. This cluster shows a strong cooling flow, with a mass accretion rate of $260 M_{\odot}$ year $^{-1}$ (Allen et al. 1995).

\section{5. $A 2254$}

In Fig. 9 we show the radio image at $1.4 \mathrm{GHz}$ of the radio halo in the cluster A2254.
The source located at the position $\operatorname{RA}(2000)=$ $17^{\mathrm{h}} 17^{\mathrm{m}} 56^{\mathrm{s}}, \operatorname{DEC}(2000)=19^{\circ} 40^{\prime} 58^{\prime \prime}$, with a flux of about $38 \mathrm{mJy}$ is an unrelated discrete source, while the diffuse radio emission is not due to a blend of individual sources. The radio structure of the halo appears quite irregular with a projected size of about $5^{\prime}(1.16 \mathrm{Mpc})$. This radio halo was also observed by Owen et al. (1999).

In Fig. 10 we show the overlay between the radio (grey scale) and the X-ray emission (contour).

Both the radio and the X-ray emission show a very clumpy and an irregular structure. As expected from numerical simulations (e.g. Schindler \& Müller 1993), the irregular X-ray structure of A2254 indicates a dynamically young cluster where different units are currently merging. It is interesting that this cluster is the most irregular cluster in the set, not only in the X-rays but also in the radio morphology. Most halos are found in very massive and hot clusters which are clusters with a very deep potential. Even if the cluster has a large surface brightness, the currently merging clumps are surely characterized by smaller mass units and shallower potentials. It is therefore remarkable and surprising that significant extended radio emission is detected in this cluster.

\section{6. $A 2744$}

In Fig. 11 we present the $1.4 \mathrm{GHz}$ radio images of the cluster A2744, at different angular resolutions. The diffuse radio emission consists of a radio halo at the cluster center, and an elongated emission in the North-East peripheral region, classified as a cluster relic. The radio halo is rather regular in shape, with a size of about $7^{\prime}(2.34 \mathrm{Mpc})$, but its brightness distribution is asymmetric, being more extended toward NW. The relic is at about $6^{\prime}$ from the cluster center and has a projected size of about $6^{\prime}(2.0 \mathrm{Mpc})$. In the image at lower resolution the relic seems to be connected to the halo through a low surface brightness bridge.

X-ray data for A2744 are available from the ROSAT PSPC. In Fig. 12 we present the X-ray image (contours) obtained from the ROSAT archive by smoothing the image with a Gaussian filter of $\sigma=30^{\prime \prime}$ over-imposed to the low resolution radio image (grey scale). The X-ray emission shows substructure, which is a possible indication of a recent merger.

The radio structure of the halo is extended in the same direction as the X-ray emission.

Govoni et al. (2001) performed an analysis to quantify the similarity between the radio and the X-ray structure for A2744 and for three other clusters (Coma, A2255, A2319) containing radio halos. A linear relation between the radio and the X-ray brightness was found in A2744.

\section{X-ray analysis}

A radial surface brightness profile was extracted from all the clusters. For A520, A1664 and A2744 the radial profile was obtained by integrating the PSPC counts over concentric annuli of $15^{\prime \prime}$, around the peak of the X-ray 

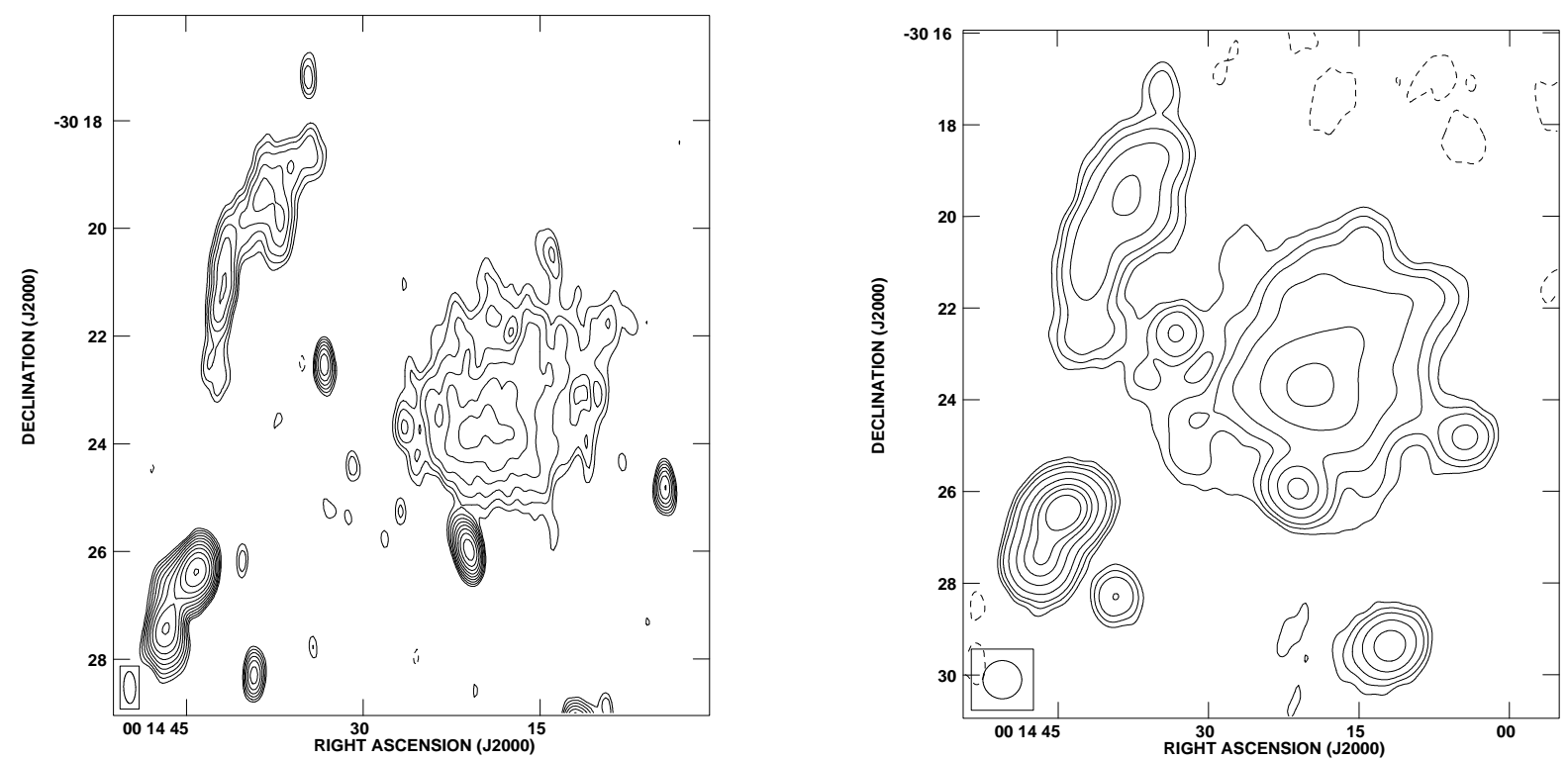

Fig. 11. Radio images at $1.4 \mathrm{GHz}$ of the halo and the relic sources in A2744. Left: the synthesized restoring beam is a Gaussian with FWHM of $36^{\prime \prime} \times 13^{\prime \prime}$. The noise level is $0.04 \mathrm{mJy} /$ beam. Contour levels are: -0.200 .200 .280 .400 .570 .801 .311 .602 .26$ $3.204 .526 .409 .0512 .8018 .1025 .6036 .20 \mathrm{mJy} /$ beam. Right: the synthesized restoring beam is a circular Gaussian with $F W H M$ of $50^{\prime \prime}$. The noise level is $0.09 \mathrm{mJy} /$ beam. Contour levels are: $-0.30 .30 .50 .81 .53571015202530 \mathrm{mJy} / \mathrm{beam}$. The elongated emission in the North-East peripheral region is a relic source.

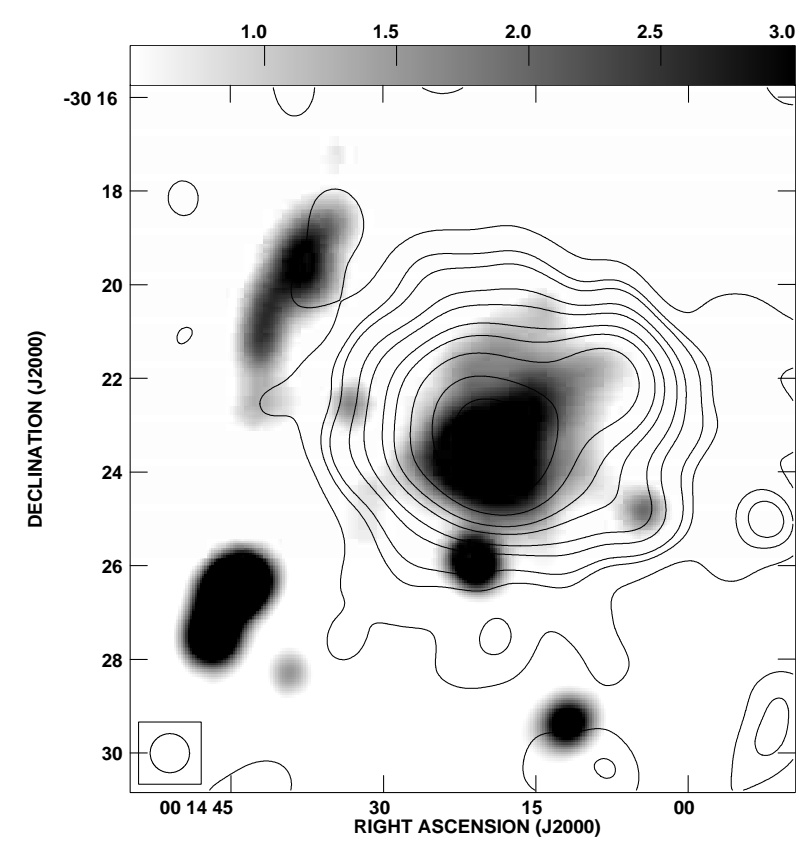

Fig. 12. Isophotal contour plots of the X-ray (PSPC) image taken from the ROSAT archive overlapped to the grey-scale image at $1.4 \mathrm{GHz}\left(F W H M 50^{\prime \prime} \times 50^{\prime \prime}\right)$ of the cluster A2744. The $\mathrm{X}$-ray contours are: 0.50 .811 .52346101520 counts/pixel $\left(1\right.$ pixel $\left.=15^{\prime \prime} \times 15^{\prime \prime}\right)$.

emission. Similarly, the radial profile of the HRI X-ray emission in A115, A773, A2254 and A1664 was obtained by integrating the photon counts over concentric annuli of $5^{\prime \prime}$.
The profiles were fitted with a hydrostatic isothermal $\beta$-model (Cavaliere \& Fusco-Femiano 1981):

$S(r)=S_{0}\left(1+r^{2} / r_{\mathrm{c}}^{2}\right)^{-3 \beta+0.5}+S_{\mathrm{b}}$

where $S_{0}$ is the central surface brightness, $r_{\mathrm{c}}$ is the core radius, and $\beta$ is the ratio of the galaxy to the gas temperature. The cluster background $S_{\mathrm{b}}$ was also fitted. The azimuthally averaged surface brightness profiles are presented in Fig. 13. The full line in each figure represents the best fit of the hydrostatic isothermal model.

The parameters of the gas distribution obtained by fitting the $\beta$-model to the surface brightness profile are reported in Table 6 . For both the PSPC and HRI images we have attempted to take substructure into account by masking out of the fit the substructure regions, the contaminating point sources and characterizing the supposedly symmetric main body of the cluster. Despite our caution in the analysis of the X-ray brightness profiles, the problem of the presence of the clumpy structure is present in most of the analyzed clusters and it is very severe in the case of A2254. For these reasons the $\beta$-model parameters for the cluster A2254 are not included in Table 6 .

In general the fit of the HRI surface brightness profile could be problematic since the X-ray cluster emission could extend over the whole field of view of the detector and the profile could not reach the background. This problem seems not to be present in the analyzed clusters, moreover, in A1664 where both the PSPC and HRI data was analyzed, the comparison of the two data set shows that the data are consistent.

Count rates, energy fluxes and X-ray luminosities are reported in Table 7. For the calculations of the X-ray 

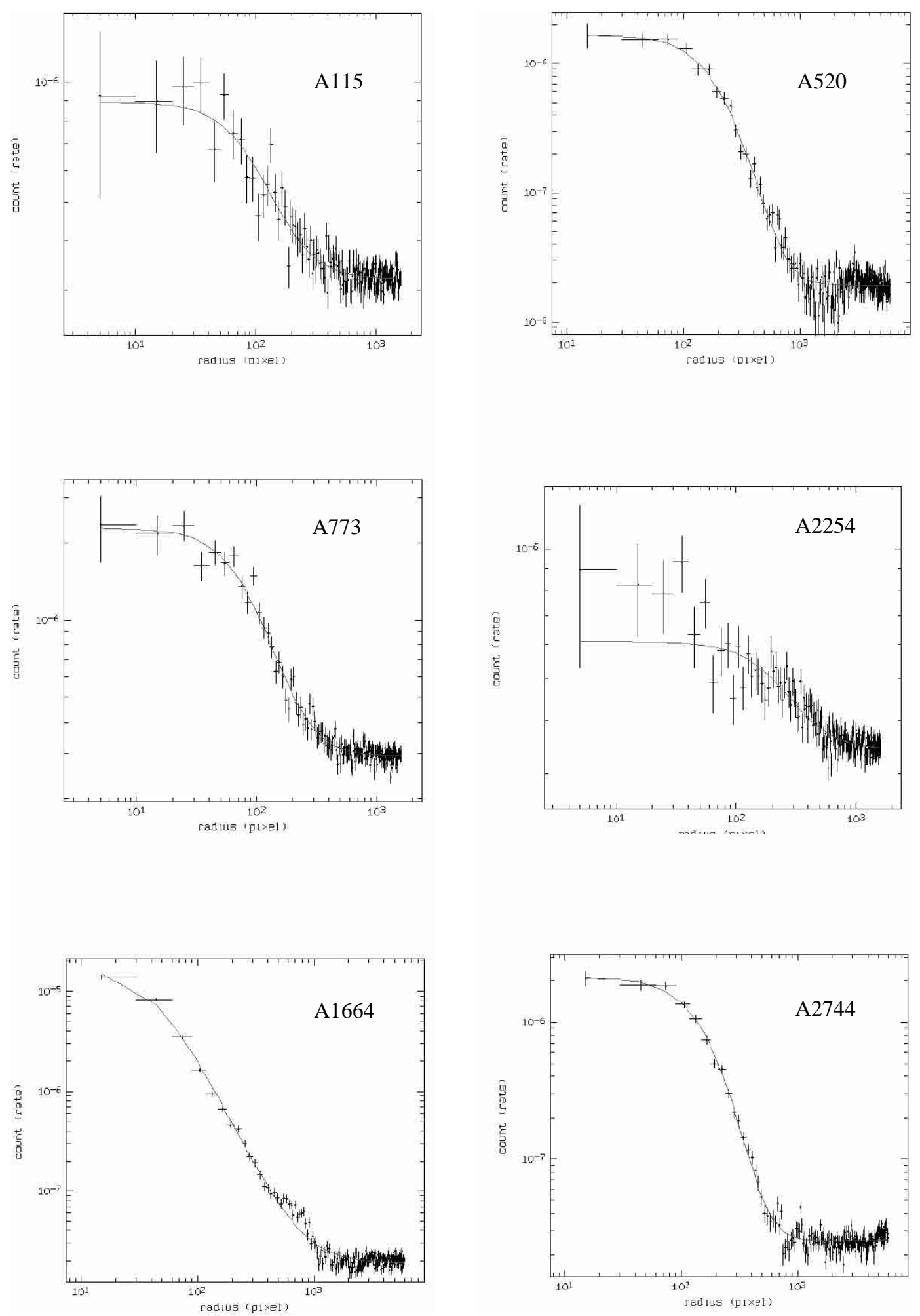

Fig. 13. Surface brightness profile of the clusters. The full lines show the best fit of the $\beta$-model.

fluxes and luminosities the cluster temperatures have been taken from the literature (when available) or from the $L_{\mathrm{X}}-T$ relation (Markevitch 1998). A quasi-bolometric luminosity, $L_{\mathrm{X}, \text { bol }}$, has been calculated in the source rest frame energy range $0.01-40.0 \mathrm{keV}$ (for the relevant range of cluster gas temperatures at least $99 \%$ of the flux is contained in this energy range).

Under the simple assumption of hydrostatic equilibrium in the ICM and spherical symmetry of the cluster, it is possible to determine the total cluster mass 

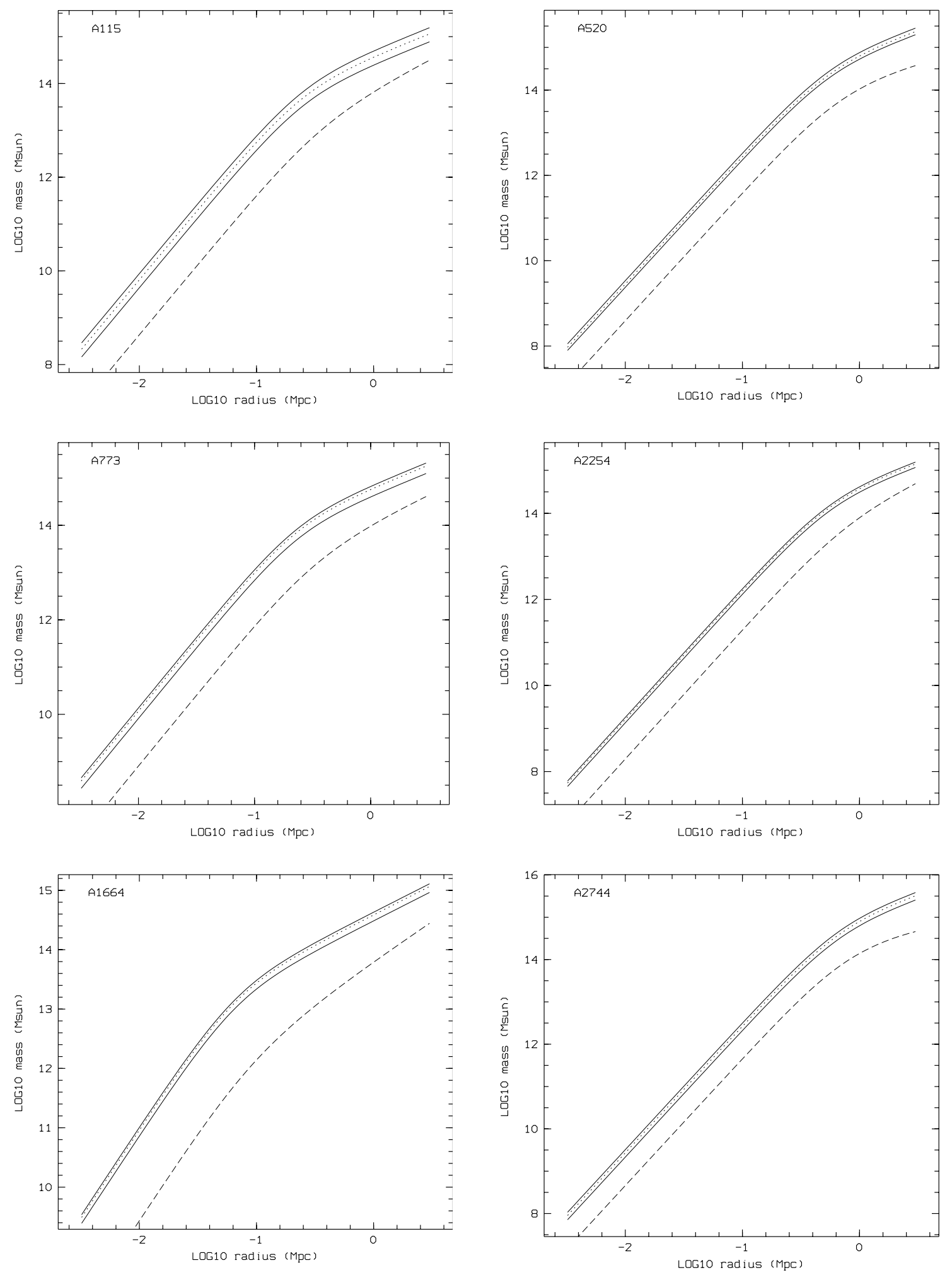

Fig. 14. Mass profile of the clusters. The dashed and dotted lines represent respectively the total gas mass of the X-ray emitting gas, and the gravitating mass, obtained by assuming hydrostatic equilibrium. Full lines around the dotted line show the uncertainty range for the gravitating mass due to the assumption of a temperature range.

from the gas density and temperature distribution. To determine the cluster mass, we use the gas density profile obtained from the $\beta$-model fit. The intracluster gas has been assumed to be isothermal at the temperature obtained from the literature. A problem in the mass computation for clusters possibly undergoing merger events could be the assumption of isothermality and hydrostatic equilibrium. Simulations of Evrard et al. (1996) and Schindler (1996) show that the mass determined in this way is in general reliable. Observationally the study 
by Finoguenov et al. (2001) shows that the assumption of isothermality in general may lead to an overestimate of the mass by about $20 \%$ at the virial radius.

Since the luminous matter in clusters is dominated by the X-ray emitting gas, the fraction $M_{\mathrm{GAS}} / M_{\mathrm{TOTAL}}$ provides a good measure of the amount of baryonic matter in the clusters. Gas fractions for nearby clusters range from about $\simeq 5$ to $30 \%$ with a positively increasing radial gradient (Böhringer 1995; Evrard 1997). Figure 14 shows the resulting mass profiles. The dashed and dotted lines represent the total gas mass of the X-ray emitting gas and the gravitating mass, respectively. Full lines show the statistical range for the gravitating mass calculated from the uncertainty in the temperature measurement.

When the inner regions of the clusters become very dense the cooling time of the gas may drop below the age of the system, and the cluster can form cooling flows. We calculated the cooling time of each cluster, at the center, and established the existence of a cooling flow when the cooling time was shorter than about $10^{10}$ years.

In the following the details of the X-ray analysis for each cluster are presented.

\section{1. $A 115$}

The computation of the azimuthally averaged profile, was obtained by masking the X-ray emission coming from the point source $3 \mathrm{C} 28$ and by excluding the southern cluster sector corresponding to the substructure. The centroid of the X-ray emission was taken approximately at the position $\mathrm{RA}(2000)=00^{\mathrm{h}} 55^{\mathrm{m}} 53.2^{\mathrm{s}}, \operatorname{DEC}(2000)=+26^{\circ} 24^{\prime} 59^{\prime \prime}$. The parameters of the best fit $\beta$-model are given in Table 6. In spite of the exclusion of the contaminating regions, the complex morphology of the cluster makes it difficult to fit the cluster with a $\beta$-model. This distorted morphology and the different performed analysis could be the reason for the difference between our $\beta$-model parameters and the parameters calculated by Shibata et al. (1999). They give the parameter values for the main peak of $\beta=1.05_{-0.06}^{+0.08}$ and $r_{\mathrm{c}}=1^{\prime} .22_{-0.24}^{+0.29}$. Their ASCA observation shows a significant temperature variation in this cluster, moreover a linking region between the main and the sub-cluster shows a high temperature compared with other regions, indicating that Abell 115 is a system in a stage of a merger event. The ASCA spectrum of the main cluster is consistent with a cooling flow model with a mass deposition rate of $89 M_{\odot} /$ yr with an upper limit of $419 M_{\odot} /$ yr. Our analysis leads to a cooling time of the same order as the Hubble time. Therefore we conclude that a faint cooling flow with a low mass-rate seems to be present in the center of A115, consistently with the ASCA results. A cooling flow could be present in this cluster since the merger has not progressed enough to disturb the cluster center.

Ikebe et al. (2001 in prep.) give a temperature of $5.9 \mathrm{keV}$ and $6.1 \mathrm{keV}$ for the Northern and the Southern peak respectively. These data are in agreement within the errors with the temperature data presented by Shibata et al. (1999). For the estimate of the X-ray luminosity, a temperature of $5.9 \mathrm{keV}$ was used. The count rate reported in Table 7 was calculated excluding the source 3C 28 but taking into account both the substructures in the X-ray emission.

For the total cluster mass determination the gas temperature was taken in the range $4-8 \mathrm{keV}$. The resultant uncertainty range for the gravitating mass profiles is presented in Fig. 14, where the gas mass profile is also shown. At a radius of $3 \mathrm{Mpc}$, the total mass of the cluster is in the range $0.77-1.5 \times 10^{15} M_{\odot}$, and the gas mass fraction is respectively in the range $17-9 \%$ which is consistent with a typical gas mass fraction in clusters.

\section{2. $A 520$}

The X-ray emission in A520 has been traced out significantly to a maximum radius of $9^{\prime}(2.3 \mathrm{Mpc})$.

The radial profile was obtained using as a centroid the X-ray peak at position $\mathrm{RA}(2000)=04^{\mathrm{h}} 54^{\mathrm{m}} 10.6^{\mathrm{s}}$, $\operatorname{DEC}(2000)=+02^{\circ} 55^{\prime} 20^{\prime \prime}$. The parameters of the best fit of a hydrostatic isothermal model are given in Table 6 .

For the calculation of the X-ray luminosity (see Table 7) a temperature of $8.3 \mathrm{keV}$ was used (Ikebe et al. 2001, in prep.).

With the gas temperature in the range $7-10 \mathrm{keV}$, the total mass of the cluster, at a radius of $3 \mathrm{Mpc}$, is in the range $1.98-2.83 \times 10^{15} M_{\odot}$, and the gas mass fraction is in the range 19-13\% (Fig. 14).

Within a radius of $300 \mathrm{kpc}$ the cooling time $(0.34 \times$ $\left.10^{11} \mathrm{yr}\right)$ is found to be longer than the Hubble time, implying the absence of a cooling flow in this cluster, in agreement with Allen \& Fabian (1998).

The absence of a cooling flow, the extended core radius and the high X-ray luminosity could indicate that A520 may be characterized by a merger event.

\section{3. $A 773$}

The X-ray emission of the cluster shows two peaks at a distance of about $30^{\prime \prime}$.

The surface brightness profile of the cluster was obtained without masking any region and by fixing the centroid of the X-ray emission between the two peaks on the approximate symmetry center in the position $\operatorname{RA}(2000)=$ $09^{\mathrm{h}} 17^{\mathrm{m}} 52.5^{\mathrm{s}}, \operatorname{DEC}(2000)=51^{\circ} 43^{\prime} 41^{\prime \prime}$.

The parameters of the $\beta$-model are given in Table 6 . These results are in agreement with the core radius and the $\beta$ obtained by Rizza et al. (1998).

To determine the total cluster mass the gas temperature was taken in the range $6-10 \mathrm{keV}$. The total cluster mass profile and the gas mass profile of A773 are presented in Fig. 14. In the figure the dotted line represent the total cluster mass considering the cluster in isothermal equilibrium with a temperature of $8.6 \mathrm{keV}$ (Ikebe et al. 2001, in prep.). 
Table 6. X-ray fits.

\begin{tabular}{cccccc}
\hline Name & $\begin{array}{c}S_{0} \\
\mathrm{Cts}_{\mathrm{s} \mathrm{arcsec}} \mathrm{arcs}^{2}\end{array}$ & $\begin{array}{c}S_{\mathrm{b}} \\
\mathrm{Cts} / \mathrm{s} \mathrm{arcsec}^{2}\end{array}$ & $\begin{array}{c}r_{\mathrm{c}} \\
\operatorname{arcsec}\end{array}$ & $\beta$ & $\begin{array}{c}n_{0} \\
\mathrm{~cm}^{-3}\end{array}$ \\
\hline A115 & $2.29 \times 10^{-06}$ & $12.86 \times 10^{-07}$ & $58_{-18}^{+37}$ & $0.59_{-0.11}^{+0.24}$ & $3.6 \times 10^{-03}$ \\
A520 & $6.64 \times 10^{-06}$ & $7.90 \times 10^{-08}$ & $127_{-16}^{+17}$ & $0.87_{-0.07}^{+0.09}$ & $3.2 \times 10^{-03}$ \\
A773 & $8.04 \times 10^{-06}$ & $11.68 \times 10^{-07}$ & $50_{-7}^{+10}$ & $0.63_{-0.05}^{+0.07}$ & $6.7 \times 10^{-03}$ \\
A1664 & $6.89 \times 10^{-05}$ & $7.65 \times 10^{-08}$ & $21_{-2}^{+2}$ & $0.55_{-0.01}^{+0.01}$ & $22.8 \times 10^{-03}$ \\
A2254 & - & - & - & - & - \\
A2744 & $8.44 \times 10^{-06}$ & $10.20 \times 10^{-08}$ & $115_{-10}^{+13}$ & $1.00_{-0.07}^{+0.09}$ & $3.8 \times 10^{-03}$ \\
\hline
\end{tabular}

Col. 1: cluster name; Col. 2: central surface brightness; Col. 3: background surface brightness;

Col. 4: core radius; Col. 5: $\beta$ parameter; Col. 6: central gas density.

Table 7. X-ray parameters.

\begin{tabular}{ccccc}
\hline Name & $\begin{array}{c}\text { count rate } \\
\mathrm{s}^{-1}\end{array}$ & $\begin{array}{c}\text { flux }(0.1-2.4 \mathrm{keV}) \\
\operatorname{erg~s}^{-1} \mathrm{~cm}^{-2}\end{array}$ & $\begin{array}{c}L_{\mathrm{X}}(0.1-2.4 \mathrm{keV}) \\
\mathrm{erg} \mathrm{s}^{-1}\end{array}$ & $\begin{array}{c}L_{\mathrm{X}, \text { bol }} \\
\mathrm{erg} \mathrm{s}^{-} 1\end{array}$ \\
\hline A115 & $0.177 \pm 0.008$ & $9.57 \times 10^{-12}$ & $1.57 \times 10^{45}$ & $3.6 \times 10^{45}$ \\
A520 & $0.309 \pm 0.009$ & $7.33 \times 10^{-12}$ & $1.22 \times 10^{45}$ & $3.4 \times 10^{45}$ \\
A773 & $0.121 \pm 0.004$ & $5.16 \times 10^{-12}$ & $1.01 \times 10^{45}$ & $2.9 \times 10^{45}$ \\
A1664 & $0.429 \pm 0.007$ & $1.05 \times 10^{-11}$ & $0.72 \times 10^{45}$ & $1.8 \times 10^{45}$ \\
A2254 & $0.105 \pm 0.005$ & $5.66 \times 10^{-12}$ & $0.76 \times 10^{45}$ & $1.9 \times 10^{45}$ \\
A2744 & $0.265 \pm 0.006$ & $5.38 \times 10^{-12}$ & $2.10 \times 10^{45}$ & $6.5 \times 10^{45}$ \\
\hline
\end{tabular}

Col. 1: cluster name; Col. 2: X-ray count rate; Col. 3: X-ray flux, calculated in the 0.1-2.4 energy band;

Col. 4: X-ray luminosity, calculated in the 0.1-2.4 energy band; Col. 5: X-ray bolometric luminosity.

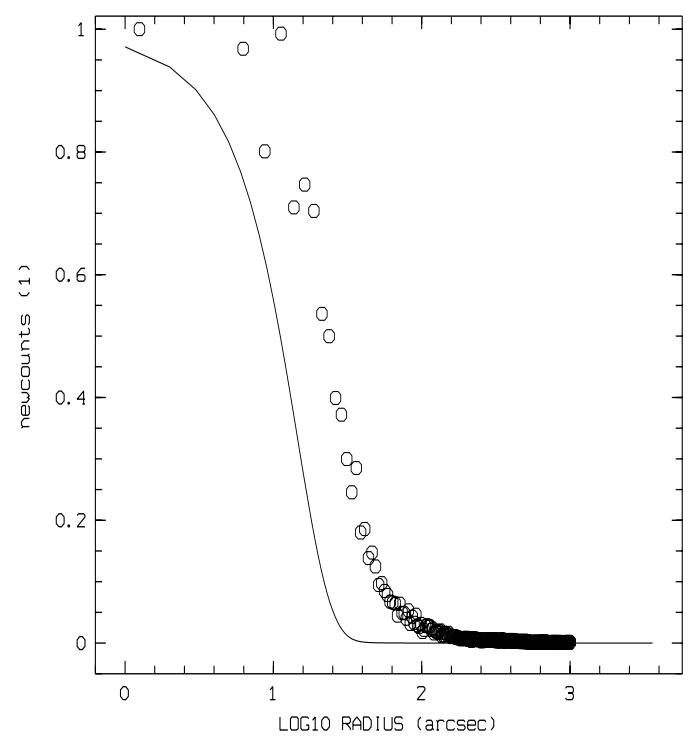

Fig. 15. Comparison of the normalized surface brightness profile (open dots) of the cluster A1664 in the PSPC image with the point spread function (PSF) of the PSPC detector (solid line).

At a radius of $3 \mathrm{Mpc}$, the total mass of the cluster is in the range $1.25-2.08 \times 10^{15} M_{\odot}$, and the gas mass fraction is in the range $32-19 \%$.

Within a radius of $300 \mathrm{kpc}$ the cooling time $(0.17 \times$ $10^{11} \mathrm{yr}$ ) is found to be only marginally larger than the Hubble time, therefore we consider that this cluster has no significant cooling flow, in agreement with Allen \& Fabian (1998).

\section{4. $A 1664$}

The X-ray emission of A1664 could be traced out significantly to a maximum radius of $11^{\prime}(2 \mathrm{Mpc})$.

The centroid of the radial profile was chosen on the maximum of the PSPC image, i.e. at $\operatorname{RA}(2000)=$ $13^{\mathrm{h}} 03^{\mathrm{m}} 43^{\mathrm{s}}, \operatorname{DEC}(2000)=-24^{\circ} 14^{\prime} 45^{\prime \prime}$. The fitting results are summarized in Table 6.

A1664 shows a very steep surface brightness profile with a strong central peak. The peaked central surface brightness could be due to a very strong cooling flow in the cluster center, or it could be the result from a central point source. To exclude the strong influence of a point source, Fig. 15 shows the comparison of the normalized surface brightness profile of the cluster in the PSPC image with the point spread function (PSF) of the PSPC detector. From the figure it is clear that the central surface brightness peak is not caused by a point source.

The total cluster mass was obtained taking the gas temperatures in the range $5-8 \mathrm{keV}$. The total cluster mass profile and the gas mass profile of A1664 are presented in Fig. 14. In the figure the dotted line represents the total cluster mass considering the cluster in isothermal equilibrium with a temperature of $6.8 \mathrm{keV}$. At a radius of $3 \mathrm{Mpc}$, the total mass of the cluster is in the range $0.92-1.47 \times 10^{15} M_{\odot}$, and the gas mass fraction is in the 
range $30-19 \%$ which is consistent with a typical gas mass fraction in cluster.

Within a radius of $300 \mathrm{kpc}$ the cooling time $(0.44 \times$ $10^{10} \mathrm{yr}$ ) was found to be smaller than the Hubble time. This result and the presence of a strong central peak in the surface brightness profile is consistent with the presence of a cooling flow in this cluster.

\subsection{A2254}

The X-ray emission of A2254 is clumpy and irregular. The surface brightness profile is therefore not well represented by a $\beta$-model. For this reason we do not give the best fit parameters.

The count rate reported in Table 7 was calculated excluding the X-ray point source visible on the East side of the X-ray image.

The total cluster mass was estimated with temperature in the range $6-8 \mathrm{keV}$, and rough $\beta$ parameters. These values should be taken cautiously, since the simple $\beta$-model appears not adequate to describe the observed X-ray brightness profile for this cluster. At a radius of $3 \mathrm{Mpc}$, the total mass of the cluster is in the range $1.8-2.4 \times 10^{15} M_{\odot}$, and the gas mass fraction is in the range $21-16 \%$ (see Fig. 14).

The cooling time is found to be longer than the Hubble time, implying the absence of a cooling flow in this cluster.

\section{6. $A 2744$}

The X-ray emission of A2744 has been traced out significantly to a maximum radius of $11^{\prime}(3.7 \mathrm{Mpc})$. The $\mathrm{X}$-ray peak is at $\mathrm{RA}(2000)=00^{\mathrm{h}} 14^{\mathrm{m}} 18.7^{\mathrm{s}}, \operatorname{DEC}(2000)=$ $-30^{\circ} 23^{\prime} 16^{\prime \prime}$. The $\mathrm{X}$-ray emission is characterized by the presence of substructure. In particular a sub-clump is located at a distance of about $150^{\prime \prime}$ from the cluster center on the North-West side.

The derived azimuthally averaged surface brightness was obtained by excluding the substructure on the northwest side. The centroid of the X-ray emission was taken in coincidence with the X-ray peak. The surface brightness profile results quite regular and well described by a $\beta$ model. The parameters obtained from the fit are presented in Table 6.

Figure 14 shows the gas mass profile and the total mass profile calculated assuming an isothermal temperature profile in the temperature range $8-12 \mathrm{keV}$. The dotted line represents the total cluster mass calculated considering a temperature of $10.1 \mathrm{keV}$ (Ikebe et al. 2001, in prep.). At a radius of $3 \mathrm{Mpc}$, the total mass of the cluster is in the range $2.55-3.82 \times 10^{15} M_{\odot}$, and the gas mass fraction is in the range $18-12 \%$ which is consistent with a typical gas mass fraction in cluster.

In agreement with Allen \& Fabian (1998), the cooling time $\left(0.31 \times 10^{11} \mathrm{yr}\right)$ of A2744 is found to be longer than the Hubble time, implying the absence of a cooling flow in this cluster.
Due to the absence of cooling flow and the presence of substructures A2744 could be a cluster with an ongoing merger event.

\section{Discussion and conclusions}

We confirm the presence of diffuse radio emissions in the six clusters analyzed here. In A520, A773, A2254 and A2744 radio halos are detected at the cluster center. In A115, A1664 and A2744 a peripheral source is present. We note that both a halo and a relic are present in A2744, like in other cases known in literature (e.g. Coma, A2255, A2256).

The halos in A520, A773 and A2744, appear extended and quite regular. On the contrary, the halo in A2254 is very clumpy and irregular. In this cluster, also the X-ray emission is quite irregular.

The presence of a diffuse radio emission in these clusters is a direct evidence for the presence of a magnetic field associated with the intergalactic medium of the cluster. Under equipartition conditions, we estimate magnetic fields of the order of $1 \mu \mathrm{G}$, permeating a large region of the clusters, up to $\sim 1 \mathrm{Mpc}$ radius.

From our data we can obtain some indications for the presence of a merger process and cooling flow in the clusters. Clumpy and unrelaxed X-ray structures can be considered as indicators of cluster mergers. In particular, the clusters A2744 and A773 could be in the process of an ongoing merger while A520 may be at a later merger state. Finally, the clumpy structure in A2254 could indicate a dynamically young cluster where the merging involves several different small units. Therefore, we confirm that the formation of the halos sources could be connected to the presence of a cluster merger process, although the stage of the merger process can be very different. We note that the clusters A2744, A520 A773 and 2254 are all noncooling flow clusters. This is expected, since simulations (McGlynn \& Fabian 1984; Roettiger et al. 1996; Gómez et al. 2001) suggest that mergers can disrupt cooling flows.

While until now there is no evidence of clusters with both radio halo and massive cooling flow, clusters with a cooling flow can host a relic source as A85 or A133. The cluster A1664 is another example. The distorted X-ray image of A1664 could indicate the presence of a recent merger, probably between the main body of A1664 and a smaller unit. This produced an asymmetric outgoing shock wave and the disturbance did not reach the cluster center with enough effect to disrupt the cooling flow. This is in agreement with the current interpretation of a relic source as the product of an outgoing merger shock wave (Enßlin et al. 1998; Roettiger et al. 1999).

Also in the cluster A115 we have evidence of a merger in a early stage and consistent with the presence of a faint cooling flow. In this cluster as discussed before a relic source have been detected.

From previous studies, some clusters were found to show a close morphological similarity between the radio and the X-ray emission (e.g. Coma, A2255, A2319). 

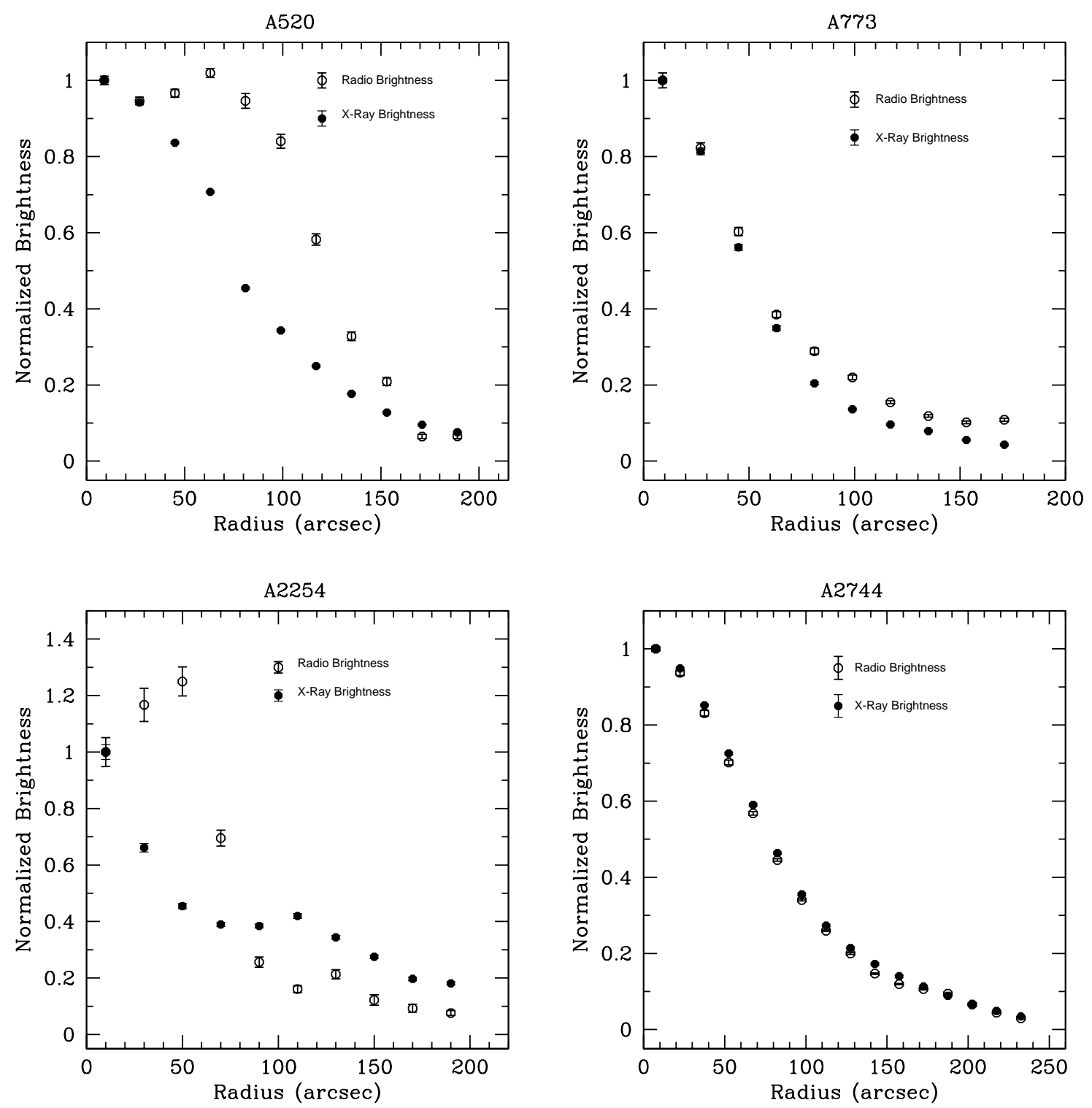

Fig. 16. Normalized radio and X-ray profile of the four clusters containing a radio halo.

In Fig. 16 we compare the shape of the normalized radio and X-ray brightness profile of the four clusters containing a radio halo. To obtain these profiles, the mean and the mean error were estimated within concentric rings centered on the X-ray symmetry center of the clusters. Point sources were excluded from the analysis. As already noted in Govoni et al. (2001), in A2744 we found a perfect similarity of the two profiles.

In A773 the two profiles are quite similar, while in A2254 and in A520 the radio and X-ray profiles show a different shape. In A2254, the different profiles reflect the fact that this cluster is very clumpy in both the radio and $\mathrm{X}$-ray emission. In A520, the halo is elongated, whereas the X-ray emission is much more extended and symmetric. Since the A520 radio profile appears flat, we cannot exclude that this source is not a real halo but a source at the cluster periphery and seen in projection onto the cluster center. This possibility should be checked in future also with polarization studies.

The radio properties of halos are linked to the properties of the X-ray emitting gas through a correlation between the halo monochromatic radio power and the X-ray luminosity (Liang et al. 2000; Feretti 1999). In Fig. 17 we present the relation, with the addition of the new data obtained in this work.

We checked if the correlation is real or induced by distance effects. Since it is still present plotting the radio flux versus the X-ray flux and considering that the slope of the correlation is steep, we conclude that it is real. 


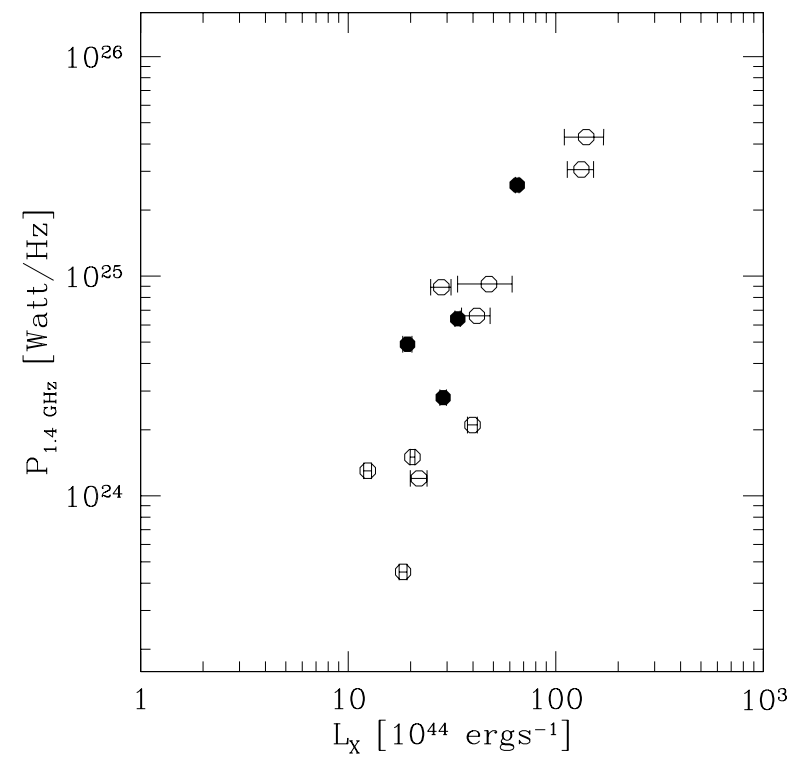

Fig. 17. Relation between the halo radio power at $1.4 \mathrm{GHz}$ and cluster X-ray bolometric luminosity. Filled and open dots represent the clusters studied in this work and the clusters taken from the literature, respectively.

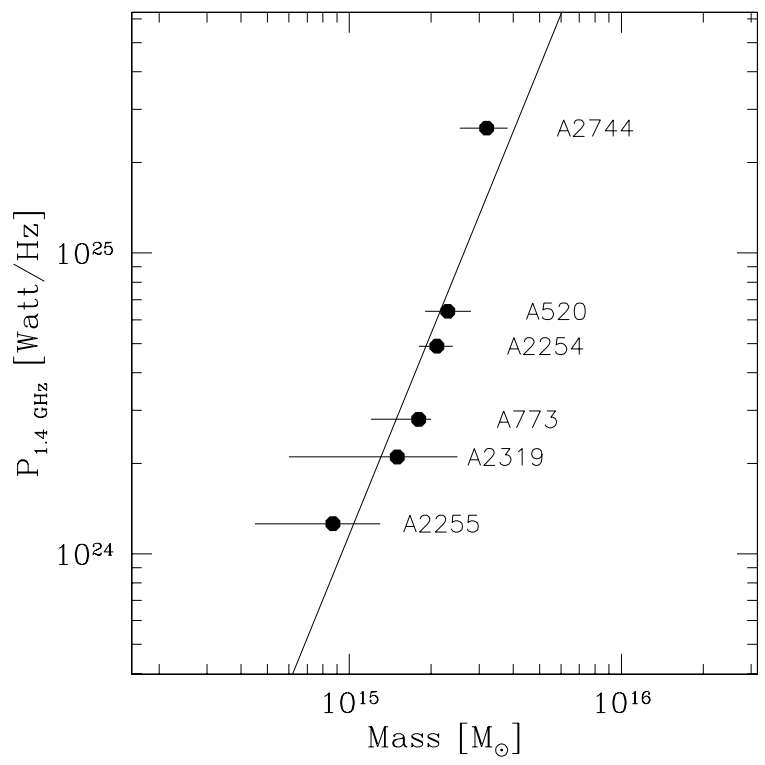

Fig. 18. Relation between the halo radio power at $1.4 \mathrm{GHz}$ and the gravitational mass for the clusters studied in this work, A2319 (Feretti et al. 1997a) and A2255 (Feretti et al. 1997b).

The clusters hosting radio halos analyzed in this work are all X-ray luminous and consequently they have a high temperature and large mass.

Since the cluster temperature (e.g., Markevitch et al. 1998) and the cluster mass (e.g., Reiprich \& Böhringer 1999) are related to the X-ray luminosity, a relation is expected also between the monochromatic radio power and the cluster temperature (Liang et al. 2000) and between the monochromatic radio power and the cluster mass.
In all the analyzed clusters, mass values larger than $1.1 \times 10^{15} M_{\odot}$ are found for the total gravitating mass within $3 \mathrm{Mpc}$. In particular, the two clusters A115 and A1664 hosting relic sources have a total mass of about $1.1 \times 10^{15}$ and $1.2 \times 10^{15} M_{\odot}$, respectively, while the mass of the clusters with radio halos range between $1.8-3.2 \times 10^{15} M_{\odot}$. The most massive cluster is A2744 which hosts both a relic and a halo source.

In Fig. 18 we plot the radio power calculated at $1.4 \mathrm{GHz}$ versus the gravitating mass (within $3 \mathrm{Mpc}$ ). There is a clear trend of increasing radio power for increasing mass. The relation is well fitted with a power law $P_{1.4 \mathrm{GHz}} \propto \mathrm{Mass}^{2.2}$.

All the points in the fit are from this work except for A2255 and A2319 whose values are taken from previous works (Feretti et al. 1997a; Feretti et al. 1997b) where the mass determination was performed in the same way as in this work.

As discussed above this relation reflects the already known relation between the radio power and X-ray luminosity, but the trend shows a small scatter. If confirmed, this trend could indicate that the real connection between the radio and the $\mathrm{X}$-ray properties of the gas is primarily related to the cluster mass rather than to the cluster temperature.

The cluster mass is a logical candidate for a fundamental parameter since the energy available to accelerate relativistic particles in a merger scales as $\simeq M^{2}$ as discussed by Buote (2001). The existence of halos in massive clusters is also consistent with their serendipitous detection in clusters observed during the attempts to detect Sunyaev-Zeldovich effect in massive high redshift clusters (Liang et al. 2000; Feretti et al. 2000).

We wish to point out that not all X-ray luminous clusters show a radio halo. Indeed, diffuse halos are present in about $30-35 \%$ of clusters with $L_{\mathrm{X}}>10^{45} \mathrm{erg} \mathrm{s}^{-1}$ (e.g. Feretti et al. 2000). Therefore, the large mass (high X-ray luminosity) of a cluster is not the only condition that ensures the existence of diffuse radio emission. Also the presence of a recent merger event in the cluster is needed for the formation and maintenance of a radio halo, as stressed by Feretti (2001) and Buote (2001). These results are in line with the recent model proposed by Brunetti et al. (2001) where the importance of the cluster dynamical history and of a recent merger event is pointed out.

Acknowledgements. F. G. acknowledges the MPE of Garching for hospitality and partial financial support. We thank the anonymous referee for helpful comments. NRAO is a facility of the National Science Foundation, operated under cooperative agreement by Associated Universities, Inc. This work was partly supported by the Italian Ministry for University and Research (MURST) and by the Italian Space Agency (ASI). This research has made use of the NASA/IPAC Extragalactic Data Base (NED) which is operated by the JPL, California Institute of Technology, under contract with the National Aeronautics and Space Administration. 


\section{References}

Abell, G. O., Corwin, H. G. Jr., \& Olowin, R. P. 1989, ApJS, 70,1

Allen, S. W., Fabian, A. C., Edge, A. C., Böhringer, H., \& White, D. A. 1995, MNRAS, 275, 741

Allen, S. W., \& Fabian, A. C. 1998, MNRAS, 297, L63

Becker, R. H., White, R. L., \& Helfand, D. J. 1995, ApJ, 450, 559

Beers, T. C., Huchra, J. P., \& Geller, M. J. 1983, ApJ, 264, 356

Bliton, M., Rizza, E., Burns, J. O., Owen, F. N., \& Ledlow, M. J. 1998, MNRAS, 301, 609

Böhringer, H. 1995, Seventeenth Texas Symp., Annals of the New York Academy of Science, ed. H. Böhringer, G. E. Morfill, \& J. E. Trümper, 759, 67

Brunetti, G., Setti, G., Feretti, L., \& Giovannini, G. 2001, MNRAS, 320, 365

Buote, D. A. 2001, ApJ, 553, L15

Carlstrom, J. E., Joy, M., \& Grego, L. 1996, ApJ, 456, L57

Cavaliere, A., \& Fusco-Femiano, R. 1981, A\&A, 100, 194

Condon, J. J., Cotton, W. D., Greisen, E. W., et al. 1998, AJ, 115,1693

Ebeling, H., Voges, W., Böhringer, H., et al. 1996, MNRAS, 281,799

Enßlin, T. A., Biermann, P. L., Klein, U., \& Kohle, S. 1998, A\&A, 332, 395

Evrard, A. E. 1997, MNRAS, 292, 289

Evrard, A. E., Metzler, C. A., \& Navarro, J. F. 1996, ApJ, 469, 494

Feretti, L., Gioia, I. M, Giovannini, G., Gregorini, L., \& Padrielli, L. 1984, A\&A, 139, 50

Feretti, L., Böhringer, H., Giovannini, G., \& Neumann, D. 1997a, A\&A, 317, 432

Feretti, L., Giovannini, G., \& Böhringer, H. 1997b, New Ast., 2, 501

Feretti, L. 1999, in Proceeding of the Ringberg workshop on Diffuse Thermal and Relativistic Plasma in Galaxy Clusters, ed. H. Böhringer, L. Feretti, \& P. Schuecker, MPE Report 271, 3

Feretti, L., Brunetti, G., Giovannini, G., Govoni, F., \& Setti, G., Constructing the Universe with Clusters of Galaxies, IAP 2000 meeting Paris, ed. F. Durret, \& D. Gerbal [astro-ph/0009346]

Feretti, L. 2001, in The Universe at low radio frequencies, Symp. IAU 199, Pune (India), in press [astro-ph/0006379]

Finoguenov, A., Reiprich, T. H., \& Böhringer, H. 2001, A\&A, 368,749

Forman, W., Bechtold, J., Blair, W., et al. 1981, ApJ, 243, L133

Giovannini, G., Feretti, L., \& Gregorini, L. 1987, A\&AS, 69, 171
Giovannini, G., Feretti, L., \& Stanghellini, C. 1991, A\&A, 252, 528

Giovannini, G., Feretti, L., Venturi, T., Kim, K.-T., \& Kronberg, P. P. 1993, ApJ, 406, 399

Giovannini, G., Tordi, M., \& Feretti, L. 1999, New Ast., 4, 141

Govoni, F., Enßlin, T. A., Feretti, L., \& Giovannini, G. 2001, A\&A, 369, 441

Gómez, P. L., Loken, C., Roettiger, K., \& Burns, J. O. 2001, ApJ, in press [astro-ph/0009465]

Gregorini, L., \& Bondi, M. 1989, A\&A, 225, 333

Green, D. A. 2000, A Catalogue of Galactic Supernova Remnants (2000 August version), Mullard Radio Astronomy Observatory, Cavendish Laboratory, Cambridge, UK (available on the World-Wide-Web at http://www.mrao.cam.ac.uk/surveys/snrs/).

Ikebe, et al. 2001, in preparation

Liang, H., Hunstead, R. W., Birkinshaw, M., \& Andreani, P. 2000, ApJ, 544, 686

Markevitch, M. 1998, ApJ, 504, 27

Markevitch, M., Forman, W. R., Sarazin, C. L., \& Vikhlinin, A. 1998, ApJ, 503, 77

McGlynn, T. A., \& Fabian, A. C. 1984, MNRAS, 208, 709

Owen, F. N., Morrison, G., \& Voges, W. 1999, in Proceeding of the Ringberg workshop on Diffuse Thermal and Relativistic Plasma in Galaxy Clusters, ed. H. Böhringer, L. Feretti, \& P. Schuecker, MPE Report 271, 9

Pfeffermann, E., Briel, U. G., Hippmann, H., et al. 1987, Proc. Society for Photo-Optical Instrumentation Engineers (SPIE), 733, 519

Proust, D., Cuevas, H., Capelato, H. V., et al. 2000, A\&A, 355, 443

Rizza, E., Burns, J. O., Ledlow, M. J., et al. 1998, MNRAS, 301,328

Reiprich, T. H., Böhringer, H. 1999, AN, 320, 296

Roettiger, K., Burns, J. O., \& Loken, C. 1996, ApJ, 473, 651

Roettiger, K., Burns, J. O., \& Stone, J. M. 1999, ApJ, 518, 603

Röttgering, H., Wieringa, M., Hunstead, R., \& Ekers, R. 1997, MNRAS, 290, 57

Shibata, R., Honda, H., Ishida, M., Ohashi, T., \& Yamashita, K. 1999, ApJ, 524, 603

Schindler, S. 1996, A\&A, 305, 756

Schindler, S., \& Müller, E. 1993, A\&A, 272, 137

Trümper, J. 1983, Adv. Space Res., 2, 241

Zimmermann, H. U., Becker, W., Belloni, T., et al. 1994, EXSAS, User's Guide, MPE Report, 244

Willson, M. A. G. 1970, MNRAS, 151, 1 